\begin{tabular}{|c|c|}
\hline Title & A erodecelerator Performance of Flare Type Membrane Inflatable V ehicle in Suborbital Reentry \\
\hline Author(s) & Takahashi, Y usuke; Ha, Dongheun; Oshima, Nobuyuki; Y amada, Kazuhiko; A be, Takashi; Suzuki, Kojiro \\
\hline Citation & $\begin{array}{l}\text { Journal of Spacecraft and Rockets, 54(5), 993-1004 } \\
\text { https://doi.org/10.2514/1.A 33682 }\end{array}$ \\
\hline Issue Date & 2017-09 \\
\hline Doc URL & http:/hdl.handle.net/2115/67154 \\
\hline Rights & (c) 2017 A merican Institute of A eronautics and A stronautics \\
\hline Type & article (author version) \\
\hline File Information & paper_smaac_aero.pdf \\
\hline
\end{tabular}

Instructions for use 


\title{
Aero-decelerator Performance of Flare-type Membrane Inflatable Vehicle in Suborbital Reentry
}

\author{
Yusuke Takahashi ${ }^{1}$, Dongheun $\mathrm{Ha}^{2}$, Nobuyuki Oshima ${ }^{3}$ \\ Hokkaido University, Kita 13 Nishi 8, Kita-ku, Sapporo, Hokkaido 060-8628, Japan \\ and \\ Kazuhiko Yamada ${ }^{4}$, Takashi $\mathrm{Abe}^{5}$ \\ Japan Aerospace Exploration Agency, 3-1-1 Yoshinodai Chuo-ku, Sagamihara, Kanagawa \\ 252-5210, Japan \\ and \\ Kojiro Suzuki ${ }^{6}$ \\ The University of Tokyo, 5-1-5 Kashiwanoha, Kashiwa, Chiba 277-8561, Japan
}

\begin{abstract}
A flight experiment of an inflatable reentry vehicle, equipped with a thin-membrane aeroshell deployed by an inflatable torus structure, was performed using a JAXA S310-41 sounding rocket. The drag coefficient history was evaluated by analyzing the acceleration of the vehicle with atmospheric density and temperature using a global reference atmospheric model. The vehicle successfully demonstrated deceleration. During the reentry flight, the position, velocity, and acceleration of the vehicle were obtained by using the Global Positioning System. The experimental drag coefficient had an almost constant value of 1.5 in the supersonic region but decreased to 1.0 in the subsonic region. In the transonic region, a steep decrease of the drag coefficient was confirmed. To study the detailed aerodynamics for the reentry vehicle, flow-field simulations were conducted with computational fluid dynamics techniques. The aerodynamic force acting on the vehicle was investigated with the measured data throughout the supersonic and subsonic regions. In the flow-field simulation, the computed result for the drag coefficient shows reasonable agreement with the experimental one. In addition, a compressible effect in front of the vehicle was seen to appear in the supersonic region and a vortex ring at the rear of the vehicle was formed in the subsonic region.
\end{abstract}

\section{Nomenclature}

$a=$ acceleration, $\mathrm{m} / \mathrm{s}^{2}$

$C=$ coefficient

$D=$ drag

$E=$ internal energy, $\mathrm{J} / \mathrm{m}^{3}$, or Young's modulus, $\mathrm{Pa}$

$\mathbf{F}_{\mathrm{A}}=$ aerodynamic force vector, $\mathrm{N}$

$\mathbf{F}_{\mathrm{B}}=$ bending force vector, $\mathrm{N}$

$\mathbf{F}_{\mathrm{E}}=$ elastic force vector, $\mathrm{N}$

$\mathbf{F}_{\mathrm{S}}=$ shear force vector, $\mathrm{N}$

$h=$ membrane thickness, $\mathrm{m}$

\footnotetext{
${ }^{1}$ Assistant Professor, Faculty of Engineering; ytakahashi@eng.hokudai.ac.jp.

${ }^{2}$ Graduate student, Graduate School of Engineering. (Currently: Korea Advanced Institute of Science and Technology, Korea.)

${ }^{3}$ Professor, Faculty of Engineering.

${ }^{4}$ Assistant Professor, Institute of Space and Astronautical Science.

${ }^{5}$ Professor, Institute of Space and Astronautical Science.

${ }^{6}$ Professor, Graduate School of Frontier Sciences.
} 


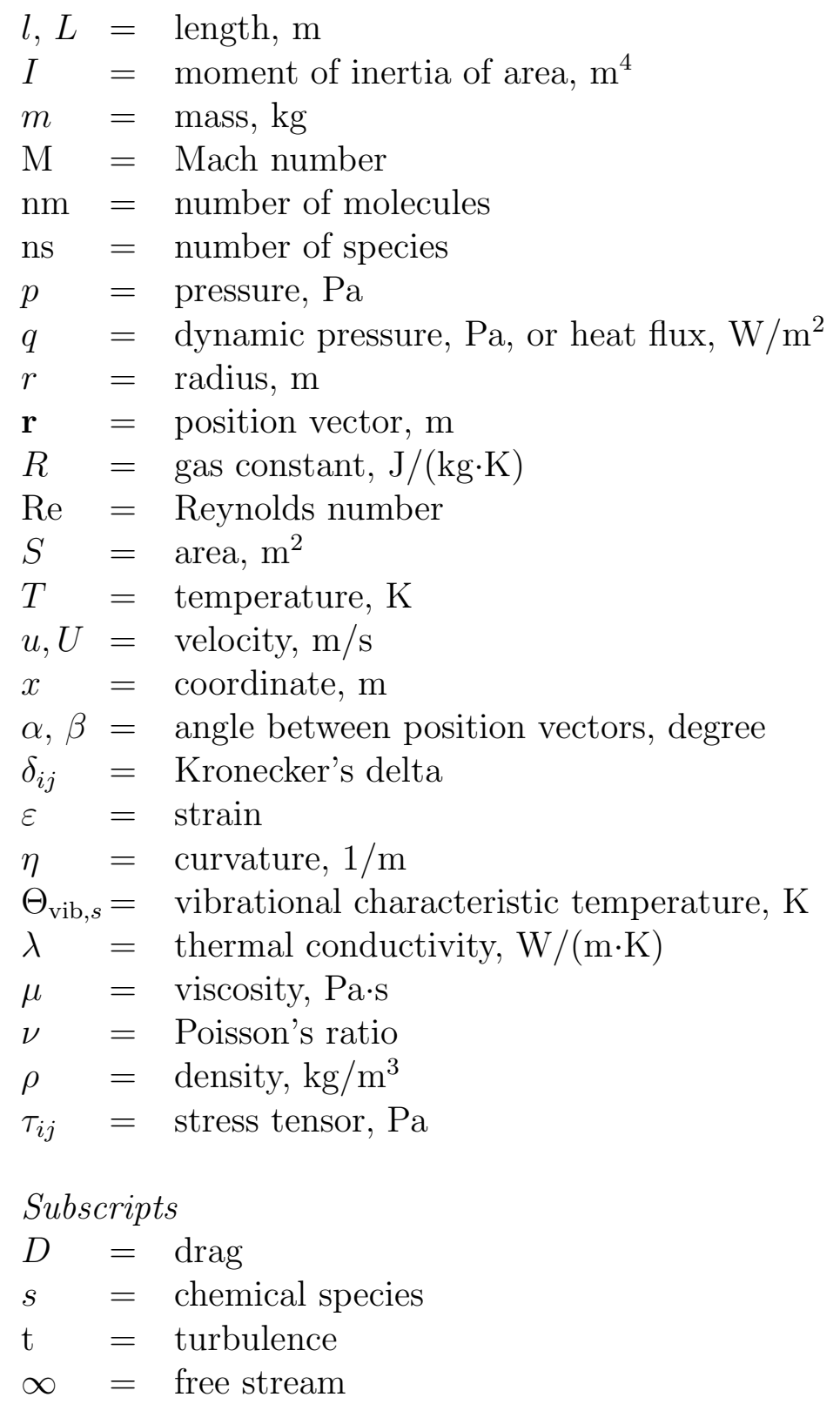

\section{Introduction}

As one of several future reentry technologies, inflatable aeroshells have been developed by the National Aeronautics and Space Administration (NASA), the European Space Agency (ESA), and the Japan Aerospace Exploration Agency (JAXA). This technology can provide several advantages, e.g., reduction in aerodynamic heating during atmospheric reentry [1-9]. For flare-type thin-membrane aeroshells, several studies of elemental technologies and demonstration flights have been performed as part of the Membrane Aeroshell for Atmospheric-entry Capsule (MAAC) project [10-14]. This type of inflatable reentry vehicle as a capsule with a tightly packed aeroshell, e.g., a slender cylindrical shape, is first transported to a given orbit. The vehicle rapidly expands the membrane aeroshell through inflation of a torus tube under vacuum and microgravity conditions on orbit. The flare-type membrane aeroshell is sustained by the inflatable torus. Because of the large area and light weight of the aeroshell, the 


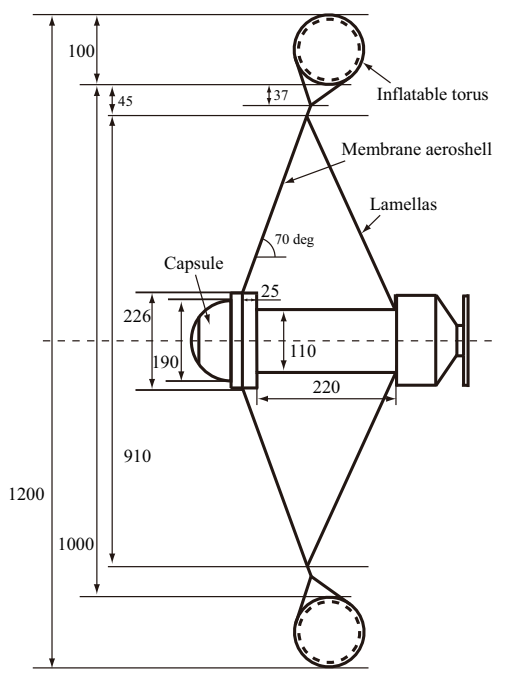

Figure 1: SMAAC configuration. (All dimensions are in millimeters.)

vehicle can achieve a low-ballistic-coefficient flight during atmospheric reentry. Additionally, because the aeroshell deployment is accomplished before deorbit, a critical operation, such as parachute extraction, is possibly dispensed with in exchange for the use of the inflatable vehicle during the entry, descent, and landing approach.

As an important milestone in the MAAC project, a reentry demonstration using an S310-41 sounding rocket (SMAAC: Sounding Rocket Experiment of Membrane Aeroshell for Atmospheric-Entry Capsule) was conducted on August 7, 2012, at the Uchinoura Space Center, Kagoshima, Japan [15-20]. This mission was a demonstration to clarify the low-ballisticcoefficient flight of a kind of inflatable vehicle during reentry. SMAAC mainly consists of three components: a capsule, a membrane aeroshell, and an inflatable torus. Figure 1 shows the configuration of SMAAC. The capsule has a semispherical configuration, with a diameter of $190 \mathrm{~mm}$. The membrane aeroshell has a flare angle of $70^{\circ}$ and a frontal projected diameter of $910 \mathrm{~mm}$ and connects to the inflatable torus. The inflatable torus has a tube diameter of $100 \mathrm{~mm}$. After being inflated, the overall diameter of the vehicle is $1200 \mathrm{~mm}$. Note that the diameter becomes $1250 \mathrm{~mm}$ when the torus is sufficiently pressurized. The primary fabric used for the aeroshell membrane and inflatable torus is Zylon, ${ }^{7}$ which has a high thermal durability and high tensile strength.

The reentry trajectory of SMAAC was evaluated by Yamada et al. [17]. After launch, the aeroshell cover of the vehicle was opened at an altitude of $100 \mathrm{~km}$, and the inflatable torus was pressurized at $106 \mathrm{~km}$ to deploy the membrane aeroshell. Then, the vehicle was separated from the rocket at an altitude of $111 \mathrm{~km}$. The vehicle started to reenter the Earth's atmosphere at an altitude of $150 \mathrm{~km}$. The vehicle had an angular velocity of $1.2 \mathrm{~Hz}$ around the body axis (in the roll direction) when separated from the rocket. The Global Reference Atmospheric Model 1999, version GRAM99 [21] (012/08/07 16:45 (JST), N29.95, E132.12), was used to evaluate the density and temperature of air during reentry. Figure 2 shows the profile for the reentry trajectory of SMAAC. The dynamic pressure rapidly increased at an altitude of $60 \mathrm{~km}$ and reached a maximum value of $500 \mathrm{~Pa}$ at an altitude of $50 \mathrm{~km}$. Then, the acceleration almost became zero below $23 \mathrm{~km}$ (500 s later after launch), and the vehicle continued to fly with a terminal velocity of $15 \mathrm{~m} / \mathrm{s}$. Finally, the vehicle splashed down and

\footnotetext{
${ }^{7}$ Toyobo Corporation; Zylon technical data: http://www.toyobo-global.com/seihin/kc/pbo/
} 
sank in the Pacific Ocean.

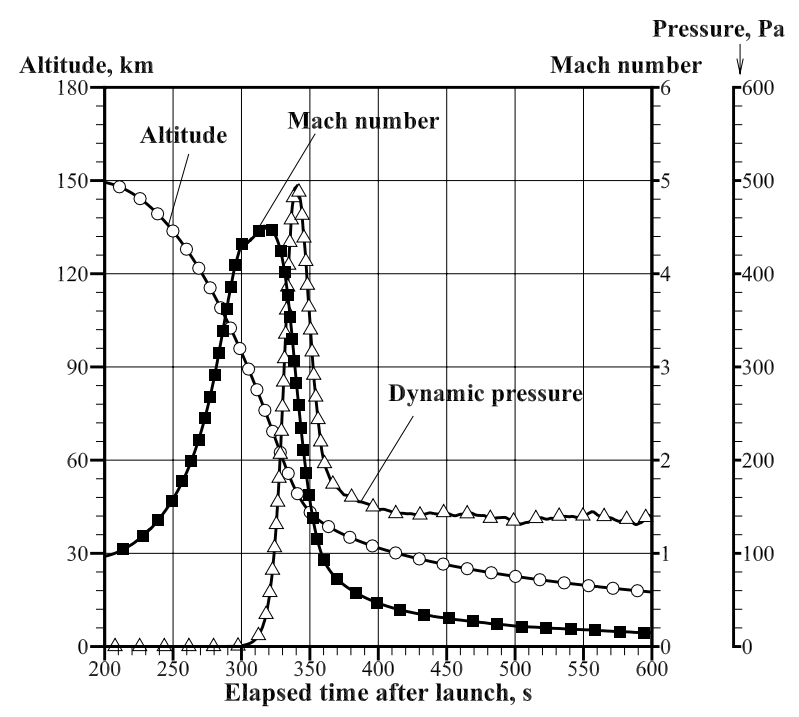

Figure 2: Trajectory profile of SMAAC.

Figure 3 shows flight profiles of the drag coefficient, which is obtained by

$$
C_{D}=\frac{m a}{q S}
$$

where $m, a, q$, and $S$ are the mass of the vehicle, its acceleration, dynamic pressure, and the front projection area of the vehicle. The acceleration and velocity of SMAAC are calculated by smoothing and subtracting position data (at a sampling rate of $10 \mathrm{~Hz}$ ) measured by the Global Positioning System (GPS) in the time direction. It was reported by Nagata et al. [19] that the SMAAC vehicle flew with an angle of attack of $<15^{\circ}$ for most altitudes considered in this figure, except for altitudes at which the Mach number $\mathrm{M}$ was $>3.5$. However, wind tunnel tests and numerical simulation approaches by Ha et al. [22] indicated that an angle of attack within $15^{\circ}$ has a small impact on the drag for a thin membrane inflatable reentry vehicle. In the flight experiment, the drag coefficient showed a nearly constant value of $\approx 1.5$ in the supersonic region. Then, a steep decrease in the transonic region appeared. The drag coefficient reached a constant value of 1.0 again and gradually decreased in the subsonic region with a decrease in Mach number. Only within the high-Mach-number regime of $\mathrm{M}>$ 3.5 is the drag coefficient low. This is mainly because the vehicle had a high angle of attack at the beginning phase of the reentry.

For the development and design of this kind of vehicle, evaluating its aerodynamics during atmospheric reentry is important. Because the reentry vehicle also experiences various flow regimes from supersonic to subsonic, it is difficult to understand the flow field solely from experimental results. Computational fluid dynamics (CFD) is a very effective tool for investigating the flow field in detail. So far, aerodynamic heating around SMAAC has been investigated using measurement results by thermocouples and the CFD technique by Takahashi et al [20]. Here, we particularly focus on the aerodynamic deceleration performance of SMAAC according to the measured drag coefficient history of the flight test and the CFD approach. In addition, the research objective also includes contributing to the construction of a flow-field simulation model for this kind of reentry vehicle. 


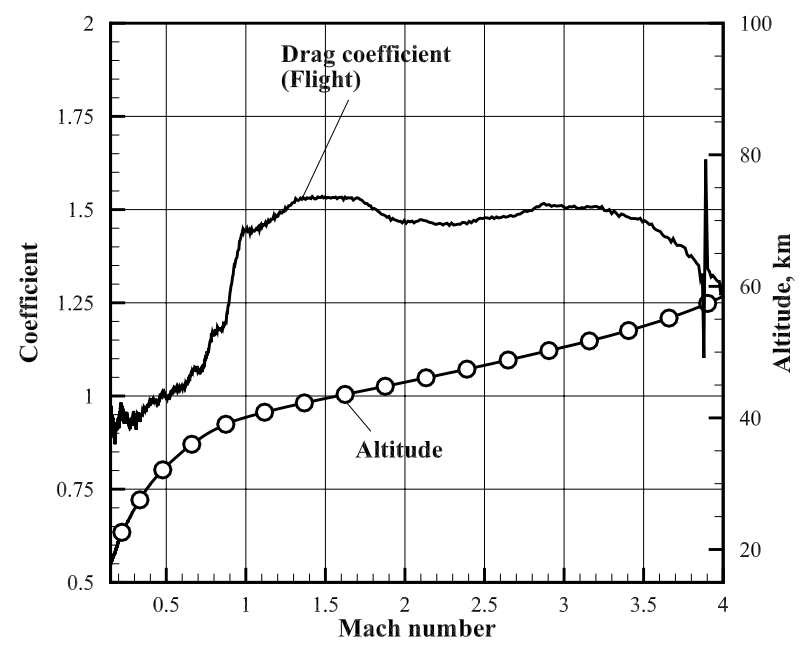

Figure 3: Drag coefficient profile obtained in the flight experiment versus Mach number.

\section{Analytic Model}

In this section, the governing equations, related physical models, and numerical method used in this study are briefly described.

\subsection{Flow Field}

The following assumptions are employed here: (1) The flow is continuum and turbulent; (2)

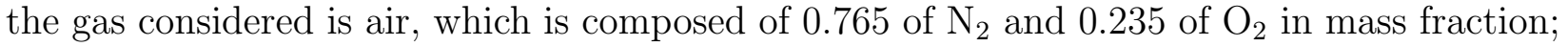
and (3) no chemical reaction occurs.

The flow field is described by the Navier-Stokes equations and the equation of state. The equations are conservation equations for the total mass, momentum, and total energy and can be expressed as follows:

$$
\begin{aligned}
& \frac{\partial \rho}{\partial t}+\frac{\partial}{\partial x_{j}}\left(\rho u_{j}\right)=0, \\
& \frac{\partial\left(\rho u_{j}\right)}{\partial t}+\frac{\partial}{\partial x_{j}}\left(\rho u_{i} u_{j}+\delta_{i j} p\right)=\frac{\partial \tau_{i j}}{\partial x_{j}}, \\
& \frac{\partial E}{\partial t}+\frac{\partial}{\partial x_{j}}\left[(E+p) u_{j}\right]=\frac{\partial}{\partial x_{j}}\left(u_{j} \tau_{i j}\right)+\frac{\partial q_{j}}{\partial x_{j}},
\end{aligned}
$$

where $\delta_{i j}$ is the Kronecker delta. Furthermore, $\tau_{i j}$ and $q_{j}$ are the stress tensor and heat flux, which are, respectively, given by

$$
\begin{aligned}
\tau_{i j} & =\left(\mu+\mu_{\mathrm{t}}\right)\left(\frac{\partial u_{i}}{\partial x_{j}}+\frac{\partial u_{j}}{\partial x_{i}}-\frac{2}{3} \frac{\partial u_{k}}{\partial x_{k}} \delta_{i j}\right), \\
q_{j} & =\left(\lambda+\lambda_{\mathrm{t}}\right) \frac{\partial T}{\partial x_{j}}
\end{aligned}
$$


The equation of state can be expressed as

$$
p=\sum_{s=1}^{\mathrm{ns}} \rho_{s} R_{s} T
$$

The total energy $E$ is given by

$$
E=\sum_{s=1}^{\mathrm{ns}} \frac{5}{2} \rho_{s} R_{s} T+\sum_{s=1}^{\mathrm{nm}} \frac{\rho_{s} R_{s} \Theta_{\mathrm{vib}, s}}{\exp \left(\Theta_{\mathrm{vib}, s} / T\right)}+\frac{1}{2} \rho u_{j} u_{j},
$$

where $\Theta_{\mathrm{vib}, s}$ is the vibrational characteristic temperature. The molecular viscosity is evaluated by using Sutherland's law. The thermal conductivity is calculated by using the viscosity and the Prandtl number, which is set to 0.9. The turbulent heat conductivity in Eq. (6) is evaluated by using the turbulent Prandtl number and the specific heat at constant pressure.

\subsection{Software Packages}

The flow field around SMAAC in the demonstration shows a wide range of changes from the supersonic to subsonic states. Numerical simulations need to be performed for various cases between these altitudes. Generally, because effective CFD algorithms for supersonic flow (compressible) and subsonic flow (incompressible) are largely different, it is desirable to use solvers suitable for the compressible and incompressible flows from the viewpoint of computational cost and accuracy. In the present study, RG-FaSTAR v2.0.0 [23] for the supersonic flow region and FrontFlow/red (FFR) Ver.3.1 [24] for subsonic flow are adopted, respectively. RG-FaSTAR is a high-enthalpy flow solver that incorporates real gas effects and thermochemical nonequilibrium and is a version of the fast unstructured CFD code FaSTAR [25], originally developed by JAXA. In contrast, FFR is a multiphysics simulation solver based on the three-dimensional incompressible Navier-Stokes (NS) equation. From the difference in their solving methods, these solvers are categorized as a density-based solver and a pressure-based solver, respectively.

Both solvers can handle unstructured grid systems including tetrahedral, triangular prism, square pyramid, and hexahedral meshes, and they have high flexibility in generating the computational grid. RG-FaSTAR and FFR include several turbulence models and are effective codes in the present study. These solvers can treat the export format of both Gridgen and Pointwise, which are programs used for grid generation. For massive parallel computation on a high-performance computer, the message passing interface (MPI) technique with a domain partition approach is adopted.

\subsubsection{RG-FaSTAR}

For RG-FaSTAR, the governing equations of the flow field are solved using a finite-volume approach. All the flow properties are set at the center of a control volume. The advection fluxes in the NS equations are calculated using the SLAU scheme [26] with the MUSCL interpolation method for high accuracy. The viscous fluxes are evaluated using the second-order central difference method. The spatial gradients of the flow properties are calculated using the Green-Gauss method. Time integration is performed using an implicit time-marching method. The system of governing equations is transformed into the delta form, and the solution is updated at each time step. For time integration, the lower-upper symmetric Gauss-Seidel (LU-SGS) method [27] is employed. For a turbulence model, the shear stress transport turbulence model 2003 version (SST2003) [28] is adopted. 


\subsubsection{FrontFlow/red}

In this simulation, for FFR, the low-Mach-number approximation is used to enable treatment of transonic flow in addition to subsonic flow. The governing equations for FFR are also solved using a finite-volume approach. To solve for the numerical fluxes, a blended scheme (of a second-order central difference method for $95 \%$ and a first-order upwind method for $5 \%$ ) is used for the advection term to decrease numerical oscillations. All the viscous terms are calculated using a second-order central difference method. The spatial gradients of flow field variables at the cell center are estimated by using the Gauss method. For the time integrations, the Crank-Nicolson implicit scheme is adopted. Pressure-velocity coupling is conducted using the simplified marker and cell (SMAC) algorithm. The Poisson equation is used to correct pressure and is solved by using the incomplete Cholesky conjugate gradient (ICCG) method. As a turbulence model, the standard Smagorinsky model (SS) [29] of the large eddy simulation (LES) is adopted to express the unsteady flow in the subsonic flow region. The Smagorinsky constant is set to 0.15 .

\subsection{Computational Conditions}

The present calculations are performed for altitudes from 58 to $1.0 \mathrm{~km}$ as listed in Table 1 . For supersonic and transonic regions at altitudes between 58.0 and $37.0 \mathrm{~km}, \mathrm{RG}-\mathrm{FaSTAR}$ is used, while FFR is used in transonic and subsonic regions at altitudes between 39.0 and 1.0 $\mathrm{km}$. Free-stream parameters, such as the free-stream velocity, density, and temperature, are given according to these flight conditions. The Reynolds number is calculated with viscosity by using Sutherland's law and the characteristic length, i.e., the SMAAC diameter of $1.2 \mathrm{~m}$, in addition to the reentry velocity and free-stream density. The angle of attack is always set to zero for all altitudes in this study

Table 1: Free-stream conditions.

\begin{tabular}{cccccc}
\hline $\begin{array}{c}\text { Altitude, } \\
\mathrm{km}\end{array}$ & $\begin{array}{c}\text { Density, } \\
\mathrm{kg} / \mathrm{m}^{3}\end{array}$ & $\begin{array}{c}\text { Temperature, } \\
\mathrm{K}\end{array}$ & $\begin{array}{c}\text { Velocity, } \\
\mathrm{m} / \mathrm{s}\end{array}$ & Reynolds number & Mach number \\
\hline 58.0 & $4.24 \times 10^{-4}$ & 248.3 & 1249.5 & $3.98 \times 10^{4}$ & 3.95 \\
54.0 & $6.95 \times 10^{-4}$ & 252.3 & 1127.6 & $5.72 \times 10^{4}$ & 3.50 \\
47.0 & $1.67 \times 10^{-3}$ & 263.0 & 749.7 & $9.01 \times 10^{4}$ & 2.30 \\
43.0 & $2.84 \times 10^{-3}$ & 258.6 & 485.7 & $1.00 \times 10^{5}$ & 1.51 \\
41.0 & $3.74 \times 10^{-3}$ & 254.6 & 364.8 & $1.01 \times 10^{5}$ & 1.14 \\
40.0 & $4.26 \times 10^{-3}$ & 252.6 & 315.2 & $9.94 \times 10^{4}$ & 0.989 \\
39.0 & $4.97 \times 10^{-3}$ & 250.1 & 276.3 & $1.03 \times 10^{5}$ & 0.871 \\
37.0 & $6.67 \times 10^{-3}$ & 245.1 & 225.8 & $1.14 \times 10^{5}$ & 0.719 \\
35.0 & $8.99 \times 10^{-3}$ & 240.3 & 189.0 & $1.31 \times 10^{5}$ & 0.608 \\
30.0 & $1.91 \times 10^{-2}$ & 229.4 & 122.1 & $1.87 \times 10^{5}$ & 0.402 \\
20.0 & $9.81 \times 10^{-2}$ & 209.6 & 53.1 & $4.50 \times 10^{5}$ & 0.183 \\
10.0 & $4.17 \times 10^{-1}$ & 242.4 & 25.6 & $8.18 \times 10^{5}$ & 0.0820 \\
5.0 & $7.11 \times 10^{-1}$ & 274.4 & 21.3 & $1.05 \times 10^{6}$ & 0.0640 \\
3.0 & $8.71 \times 10^{-1}$ & 285.0 & 18.4 & $1.08 \times 10^{6}$ & 0.0543 \\
1.0 & 1.06 & 295.6 & 16.5 & $1.14 \times 10^{6}$ & 0.0477 \\
\hline \hline
\end{tabular}




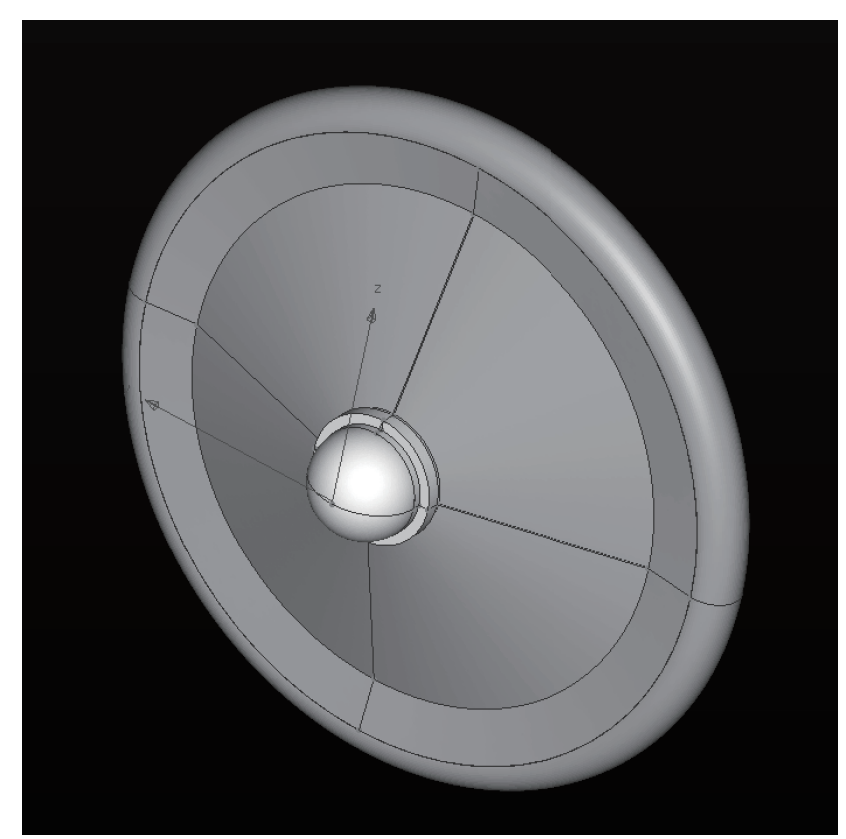

(a) Front-side view

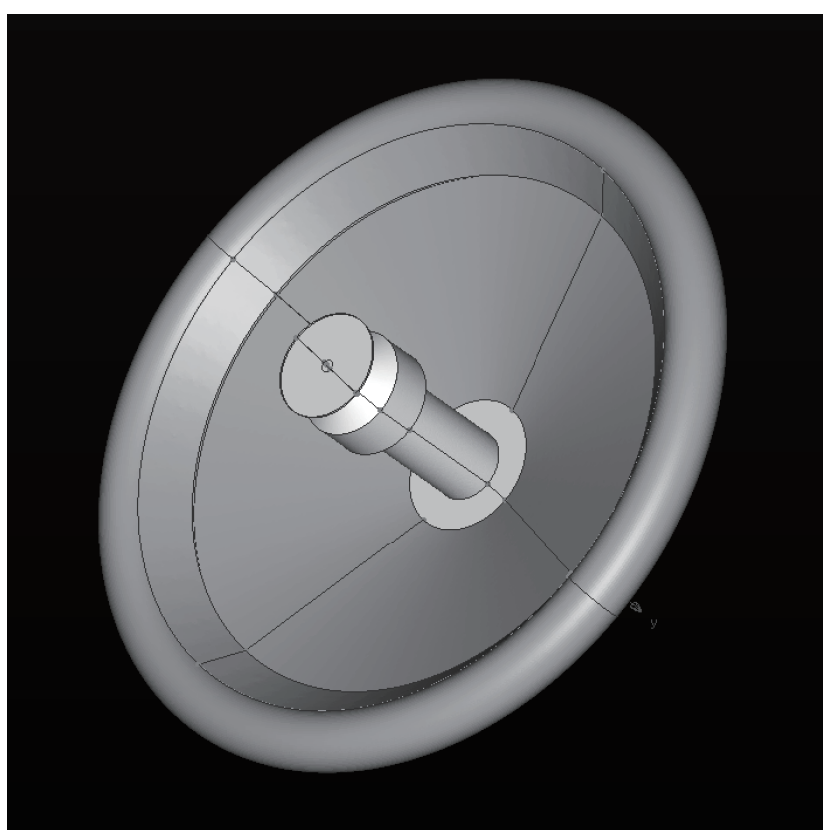

(b) Rear-side view

Figure 4: Analytic model configuration of SMAAC in RG-FaSTAR and FFR.

Figure 4 shows an analytical configuration of SMAAC. The membrane aeroshell, inflatable torus, and capsule of SMAAC are reproduced in detail as a computational model, although lamellas at the rear are not added. The configuration is assumed to be rigid, and membrane deformation of the aeroshell is not considered for the baseline case. However, for several cases, aerodynamic simulations with a membrane deformation model for the aeroshell are performed to examine aerodynamic characteristics between deformation and nondeformation (rigid case).

For the supersonic condition, the computational domain and grid system shown in Figs. 5(a) and 5(b) are used. The numbers of cells and nodes are 20,010,546 and 3,969,943, respectively, at altitudes from 58 to $47 \mathrm{~km}$. Most of the computational elements and cells in this domain are tetrahedral. By using unstructured grids, the human cost to generate the computational mesh can be drastically reduced, compared with grid generation by using structural grids such as all hexahedral meshes. However, to accurately resolve the shock wave in front of the vehicle, a hexahedral mesh is partly inserted. Square pyramid meshes are attached onto the hexahedral mesh block to connect the hexahedral and tetrahedral meshes. The numbers of cells and nodes of the computational grid used between altitudes of 43 and $37 \mathrm{~km}$ are 24,859,408 and 4,197,030, respectively. In this grid system, only a tetrahedral mesh is used throughout the whole computational domain. For boundary conditions, at the inflow, the free-stream parameters are given according to the trajectory data as listed in Table 1. The nonslip condition for the velocity is imposed at the vehicle surfaces. No pressure gradient normal to the surface is assumed. The temperature is fixed to $273 \mathrm{~K}$ at the vehicle surface. At the outlet boundary, the zeroth extrapolation condition for the flow parameters is imposed.

The computational domain and grid system for conditions in the subsonic region are shown in Figs. 6(a) and 6(b), respectively. The numbers of cells and nodes of the grid used for an altitude of $10 \mathrm{~km}$ are 31,991,401 and 5,407,191, respectively. Tetrahedral meshes are adopted throughout the whole computational domain except in a region near the outlet boundary 


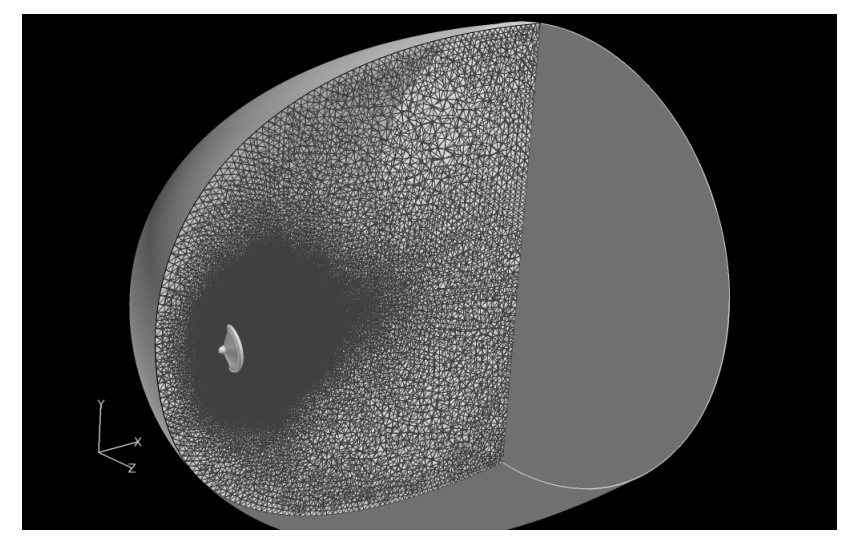

(a) Computational domain

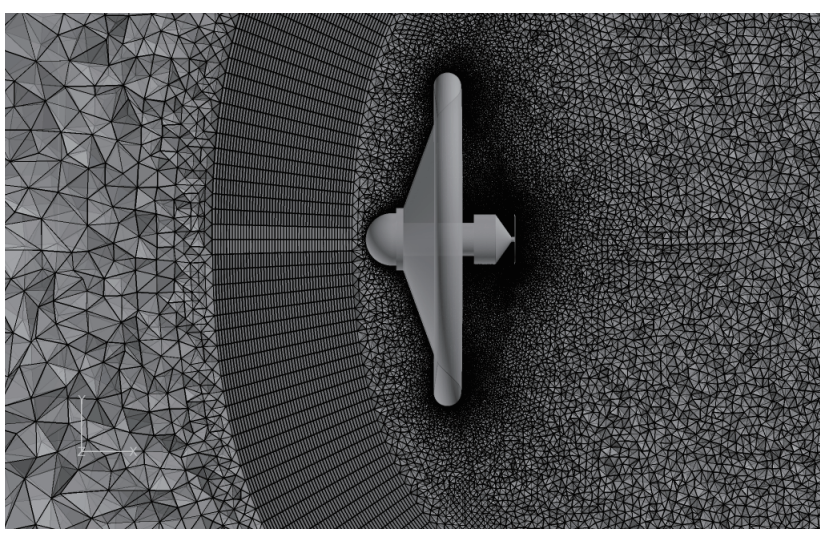

(b) Computational grid system

Figure 5: Computational domain and grid system for SMAAC at altitude of $54 \mathrm{~km}$ (RGFaSTAR).

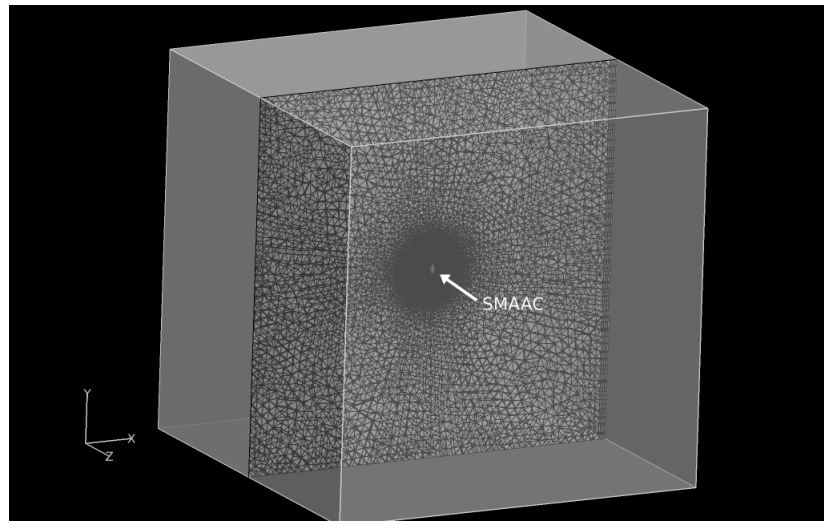

(a) Computational domain

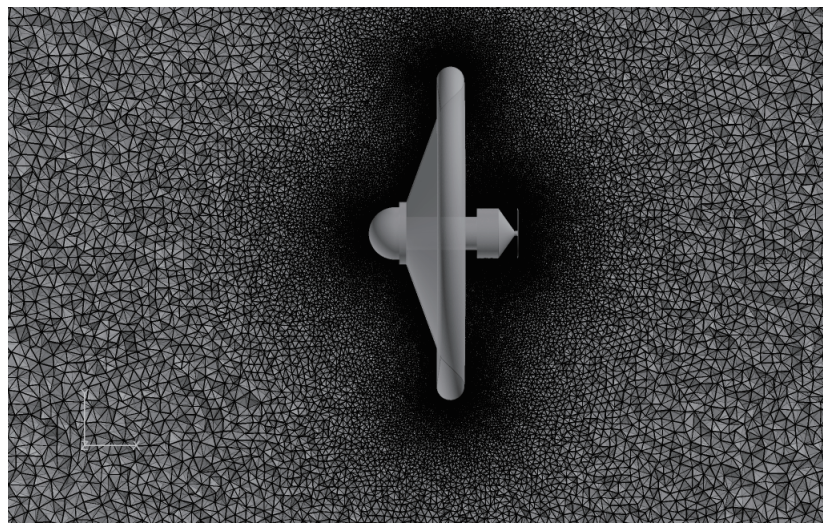

(b) Computational grid system

Figure 6: Computational domain and grid system for SMAAC at altitude of $10 \mathrm{~km}$ (FFR).

where triangular prism meshes are used to stabilize the outlet condition. In a similar fashion with the case of RG-FaSTAR, at the inflow boundary, the free-stream parameters are given according to the trajectory data. The log-law condition for the velocity is imposed at the surfaces and no pressure gradient normal to the wall is assumed.

\subsection{Membrane Deformation}

A membrane aeroshell generally deforms as a result of the aerodynamic force during atmospheric flight [30]. In the present paper, this membrane deformation is expressed using a particle-based model. This model is a three-dimensional version of the membrane model reported by Takahashi et al [20], with bending and shear effects added. It is assumed that the membrane consists of virtual particles, with springs connecting the particles. The displacement of particles from their initial state is determined by the aerodynamic force and interactions between neighboring particles as shown in Fig. 7. The virtual particle position, $\mathbf{r}$, can be described using the following equation of motion:

$$
\rho h_{0} S_{0} \frac{d^{2} \mathbf{r}}{d t^{2}}=\mathbf{F}_{\mathrm{E}}+\mathbf{F}_{\mathrm{A}}
$$


where the elastic force $\mathbf{F}_{\mathrm{E}}$ is composed of the tension force $\mathbf{F}_{\mathrm{T}}$, the shear force $\mathbf{F}_{\mathrm{S}}$, and the bending force $\mathbf{F}_{\mathrm{B}}$ as follows:

$$
\mathbf{F}_{\mathrm{E}}=\mathbf{F}_{\mathrm{T}}+\mathbf{F}_{\mathrm{S}}+\mathbf{F}_{\mathrm{B}}
$$

and $\mathbf{F}_{\mathrm{A}}$ is the aerodynamic force.

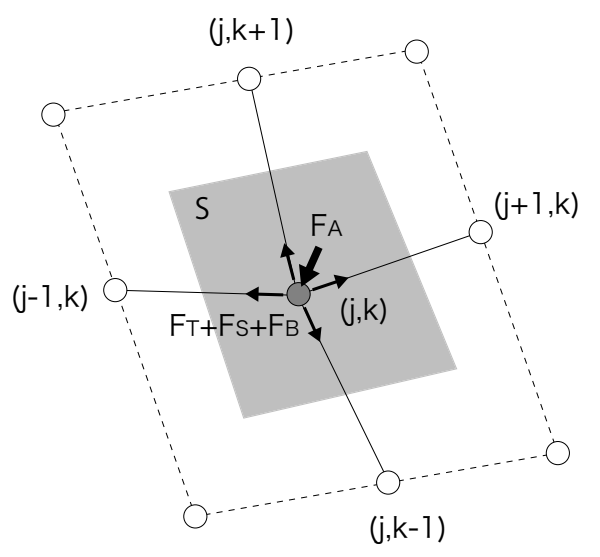

Figure 7: Schematic of the virtual particle network.

\subsubsection{Tension force}

The $(j+1 / 2, k)$ th component of the tension force $\mathbf{F}_{\mathrm{T}}$ is given by

$$
F_{\mathrm{T}\left(j+\frac{1}{2}, k\right)}=E h_{\left(j+\frac{1}{2}, k\right)} l_{\left(j+\frac{1}{2}, k\right)} \frac{\varepsilon_{j\left(j+\frac{1}{2}, k\right)}+\nu \varepsilon_{k\left(j+\frac{1}{2}, k\right)}}{1-\nu^{2}},
$$

where $E$ and $\nu$ are Young's modulus and Poisson's ratio, respectively. Strains $\varepsilon$ are expressed with length $l_{j}$ and initial length $l_{j 0}$ between virtual particles as

$$
\begin{aligned}
\varepsilon_{j\left(j+\frac{1}{2}, k\right)} & =\frac{l_{j\left(j+\frac{1}{2}, k\right)}-l_{j 0\left(j+\frac{1}{2}, k\right)}}{l_{j 0\left(j+\frac{1}{2}, k\right)}}, \\
\varepsilon_{k\left(j+\frac{1}{2}, k\right)} & =\frac{l_{k\left(j+\frac{1}{2}, k\right)}-l_{k 0\left(j+\frac{1}{2}, k\right)}}{l_{k 0\left(j+\frac{1}{2}, k\right)}} .
\end{aligned}
$$

It is assumed that no compression force works between the particles in this model, so

$$
\varepsilon_{j\left(j+\frac{1}{2}, k\right)}=0, \text { if } l_{j\left(j+\frac{1}{2}, k\right)}<l_{j 0\left(j+\frac{1}{2}, k\right)} .
$$

The tension force acting on a particle $(j, k)$ is obtained by

$$
F_{\mathrm{T}(j, k)}=F_{\mathrm{T}\left(j+\frac{1}{2}, k\right)}+F_{\mathrm{T}\left(j-\frac{1}{2}, k\right)}+F_{\mathrm{T}\left(j, k+\frac{1}{2}\right)}+F_{\mathrm{T}\left(j, k-\frac{1}{2}\right)} .
$$

\subsubsection{Shear force}

The shear force $\mathbf{F}_{\mathrm{S}}$ is evaluated as the following expression:

$$
F_{\mathrm{S}(j+, k)}=\frac{E h_{\left(j+\frac{1}{2}, k\right)} l_{j\left(j+\frac{1}{2}, k\right)}}{2(1+\nu)}\left(\frac{\gamma_{(j+, k+)}+\gamma_{(j+, k-)}}{2}\right),
$$


where $\gamma_{(j+, k+)}$ and $\gamma_{(j+, k-)}$ are the shear strains. These are obtained by using an angle $\beta_{(j+, k+)}$ between position vectors formed by particles $(j, k)-(j+1, k)$ and particles $(j, k)-(j, k+1)$ and an angle $\beta_{(j+, k-)}$ between position vectors formed by particles $(j, k)-(j+1, k)$ and particles $(j, k)-(j, k-1)$ as follows:

$$
\begin{gathered}
\gamma_{(j+, k+)}=\beta_{(j+, k+)}-\beta_{0(j+, k+)}, \\
\gamma_{(j+, k-)}=\beta_{(j+, k-)}-\beta_{0(j+, k-)} .
\end{gathered}
$$

The subscript " 0 " of angle $\beta$ in the above expressions represents the initial state in which no force acts on the particles. The shear force acting on a particle $(j, k)$ is given by

$$
F_{\mathrm{S}(j, k)}=F_{\mathrm{S}(j+, k)}+F_{\mathrm{S}(j-, k)}+F_{\mathrm{S}(j, k+)}+F_{\mathrm{S}(j, k-)} .
$$

\subsubsection{Bending force}

The bending force $\mathbf{F}_{\mathrm{B}}$ is obtained from the bending moment as follows:

$$
\begin{aligned}
& F_{\mathrm{B}(j+, k)}=\frac{M_{j(j, k)}}{l_{j\left(j+\frac{1}{2}, k\right)}}, \\
& F_{\mathrm{B}(j-, k)}=\frac{M_{j(j, k)}}{l_{j\left(j-\frac{1}{2}, k\right)}} .
\end{aligned}
$$

The bending moment is calculated by using

$$
M_{j(j, k)}=E I_{j(j, k)} \frac{\Delta \eta_{j(j, k)}+\nu \Delta \eta_{k(j, k)}}{1-\nu^{2}}
$$

where $\Delta \eta$ is given by

$$
\Delta \eta_{j(j, k)}=\eta_{j(j, k)}-\eta_{j 0(j, k)}
$$

where $\eta_{j(j, k)}$ is the curvature of neighboring particles $(j+1, k),(j, k),(j-1, k)$, and $\eta_{j 0(j, k)}$ is its initial curvature. The curvature $\eta_{j(j, k)}$ is given by the angle $\alpha_{j, k}$ between position vectors formed by particles $(j+1, k)-(j, k)$ and particles $(j-1, k)-(j, k)$ as follows:

$$
\eta_{j(j, k)}=\frac{2 \sqrt{1-\cos ^{2} \alpha_{j(j, k)}}}{\sqrt{l_{j\left(j+\frac{1}{2}, k\right)}^{2}+l_{j\left(j-\frac{1}{2}, k\right)}^{2}-2 l_{j\left(j+\frac{1}{2}, k\right)} l_{j\left(j-\frac{1}{2}, k\right)} \cos \alpha_{j(j, k)}}} .
$$

The area moment of inertia, $I_{j(j, k)}$, is calculated by using

$$
I_{j(j, k)}=\frac{l_{k(j, k)} h_{(j, k)}^{3}}{12} .
$$

The bending force acting on a particle $(j, k)$ is given as follows:

$$
F_{\mathrm{B}(j, k)}=F_{\mathrm{B}(j+, k)}+F_{\mathrm{B}(j-, k)}+F_{\mathrm{B}(j, k+)}+F_{\mathrm{B}(j, k-)} .
$$

The bending force acts in the direction normal to the membrane, in contrast to the tension and shear forces. Thus, a buckling mode can arise in the out-of-plane direction when a strong compression force is exerted in the in-plane direction. 


\subsubsection{Aerodynamic force}

The aerodynamic force $\mathbf{F}_{\mathrm{A}}$ acting on particle $(j, k)$ is simply given by

$$
F_{\mathrm{A}(j, k)}=p_{(j, k)} S_{(j, k)} .
$$

The effect of the friction force acting on the membrane surface is neglected here.

\subsubsection{Material Properties}

Young's modulus $E$ and Poisson's ratio $\nu$ of Zylon are set to $30 \mathrm{MPa}$ and 0.3 , respectively. These parameters are tuned so that the stretch of the aeroshell because of the membrane deformation corresponds to the flight experimental results, which were constructed based on images taken by onboard JPEG cameras mounted on the SMAAC's backside [17]. Thus, the textile properties of the aeroshell are assumed to be isotropic. The density of the membrane material is set to $900 \mathrm{~kg} / \mathrm{m}^{3}$. The parameter $C_{\mathrm{AE}}$, which represents the ratio of the aerodynamic force to the elastic force of the membrane, is defined by

$$
C_{\mathrm{AE}}=\frac{\rho_{\infty} U_{\infty}^{2} L}{E h_{0}},
$$

where the thickness of the Zylon fabric, $h_{0}$, is $0.155 \mathrm{~mm}$. Thus, the $C_{\mathrm{AE}}$ value of the SMAAC is on the order of $10^{-1}$ during atmospheric reentry.

\subsubsection{Implementation}

The equation of motion is solved using the fourth-order Runge-Kutta method. As the boundary, the displacement is fixed on the capsule-side end. The free-end condition on the side of the torus is set in the $x$ direction, and the fixed-end condition is set in the $y$ and $z$ directions. The flow field is simulated as weakly coupled with the membrane deformation.

\section{Rigid Model Results}

For baseline cases, aerodynamic characteristics around the SMAAC vehicle during reentry are investigated with a rigid model.

\subsection{Grid Study}

To investigate the independency of the computational grids used in this study, studies are performed for the cases of altitudes of $43 \mathrm{~km}$ in the supersonic region and $10 \mathrm{~km}$ in the subsonic region. We use fine computational grids with 41,509,051 cells and 7,008,302 nodes for $43 \mathrm{~km}$ and 52,819,386 cells and 8,884,290 nodes for $10 \mathrm{~km}$. Comparisons of the computed SMAAC drags for these altitudes are listed in Table 2. The relative errors in the drags for the two cases using the present and fine grids are within $1.3 \%$. These results indicate that the present computational grids used in the aerodynamic force prediction for SMAAC are sufficiently solved. 
Table 2: Drag force comparison for SMAAC at altitudes of 43 and $10 \mathrm{~km}$ between the present and fine grids.

\begin{tabular}{cccc}
\hline \hline Altitude, $\mathrm{km}$ & Drag (present), $\mathrm{N}$ & Drag (fine), $\mathrm{N}$ & Error, $\%$ \\
\hline 43.0 & 585.2 & 592.9 & 1.30 \\
10.0 & 128.9 & 129.6 & 0.338 \\
\hline \hline
\end{tabular}

\subsection{Drag Coefficient History}

The drag coefficient is an important parameter for aerodynamic decelerator systems. Accurate prediction of its performance has a large influence on the design and development phase of future inflatable reentry vehicles, in addition to clarifying the flow-field structure in detail as a post-flight analysis. In the present simulation, the drag $D$ is evaluated by integrating the surface pressure and the friction stress of the vehicle in the main-stream direction $(x$ direction). Drags evaluated by RG-FaSTAR using RANS-SST2003 and FFR using LES-SS as a turbulence model are steady-state and time-averaged aerodynamic forces, respectively. The predicted drag coefficient is calculated as follows:

$$
C_{D}=\frac{D}{\frac{1}{2} \rho_{\infty} U_{\infty}^{2} S}
$$

where $\rho_{\infty}$ and $U_{\infty}$ are the free-stream density and velocity, respectively. In addition, the characteristic area of SMAAC, $S$, is set to $1.13 \mathrm{~m}^{2}\left(S=\pi r^{2}\right.$, where $\left.r=0.6 \mathrm{~m}\right)$.

Figure 8 shows a comparison of computed and measured drag coefficients against Mach number. In the supersonic flow regime, the drag coefficient calculated by RG-FaSTAR is predicted to be a constant value of $C_{D}=1.5$, similar to the results of the flight test. Yamada et al. [16] reported that the drag coefficient profile has an error of approximately $5 \%$ in the supersonic regime considering a $1 \sigma$ dispersion of the atmospheric density and ambient temperature. The predicted history of the drag coefficient generally shows good agreement with the measured one. In the transonic flow region, the drag coefficient obtained by measured flight data steeply decreases. The analytic models of both solvers well describe its steep trend in the transonic region. Computational results by FFR qualitatively also show agreement with the measured data in the subsonic region.

It is found that the friction stress contribution on the surface boundary of SMAAC is markedly lower compared with that of the pressure force. For example, ratio of the friction force to the pressure force at an altitude of $30 \mathrm{~km}$ is within $0.5 \%$. The flow separates from the inflatable torus surface, and then a large recirculation region behind the vehicle is generated for all cases considered here. Thus, most of the drag force is formed by the large discrepancies between surface pressures at its front and rear.

\subsection{Supersonic Flow Region}

Figures 9(a) and 9(b) show distributions of the pressure coefficient around SMAAC at altitudes of 47 and $43 \mathrm{~km}$, respectively. The pressure coefficient is expressed by

$$
C_{p}=\frac{p-p_{\infty}}{\frac{1}{2} \rho_{\infty} U_{\infty}^{2}},
$$




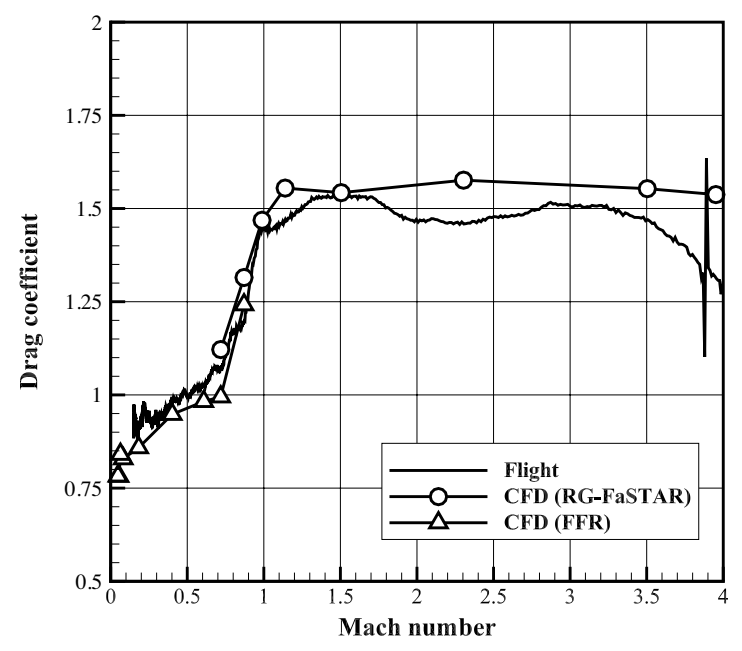

Figure 8: Comparison of drag coefficient between flight data and CFD results.

where $p_{\infty}$ is the free-stream pressure. The Mach number distributions at altitudes of 47 and $43 \mathrm{~km}$ are shown in Figs. 10(a) and 10(b), respectively. The shock wave is sharply captured in front of SMAAC at the two altitudes. In contrast, an expansion region appears to the rear of SMAAC. The pressure coefficient in the shock layer is $>1.5$ because of the effect of compressibility in the supersonic region. This value becomes high as the free-stream Mach number becomes high. The thickness of the shock layer at $43 \mathrm{~km}$ becomes larger compared with that at $47 \mathrm{~km}$ with a decrease in the free-stream Mach number. The compressed gas in the shock-layer-passing vicinity of the inflatable torus expands rearward of the vehicle. Strong expansion near the inflatable torus appears for both altitude cases of 47 and $43 \mathrm{~km}$. The flow is accelerated and a vortex ring occurs in the rear with generation of the expanded flow. However, because the pressure in the rear region is relatively low compared with that at the vehicle's front, the contribution of the wake flow to the drag force acting on SMAAC is found to be low at the higher altitude.
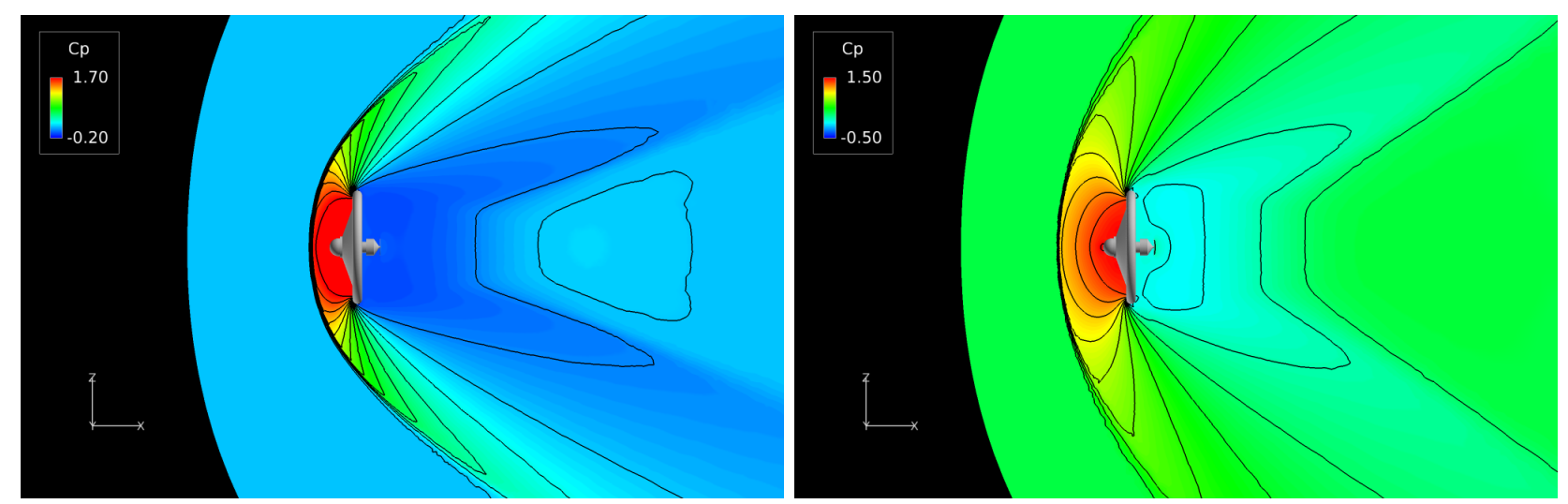

(a) Altitude of $47 \mathrm{~km}\left(\mathrm{Re}=9.01 \times 10^{4}\right.$ and $\left.\mathrm{M}=2.30\right)(\mathrm{b})$ Altitude of $43 \mathrm{~km}\left(\mathrm{Re}=1.00 \times 10^{5}\right.$ and $\left.\mathrm{M}=1.51\right)$.

Figure 9: Pressure coefficient distribution around SMAAC in the supersonic region. 

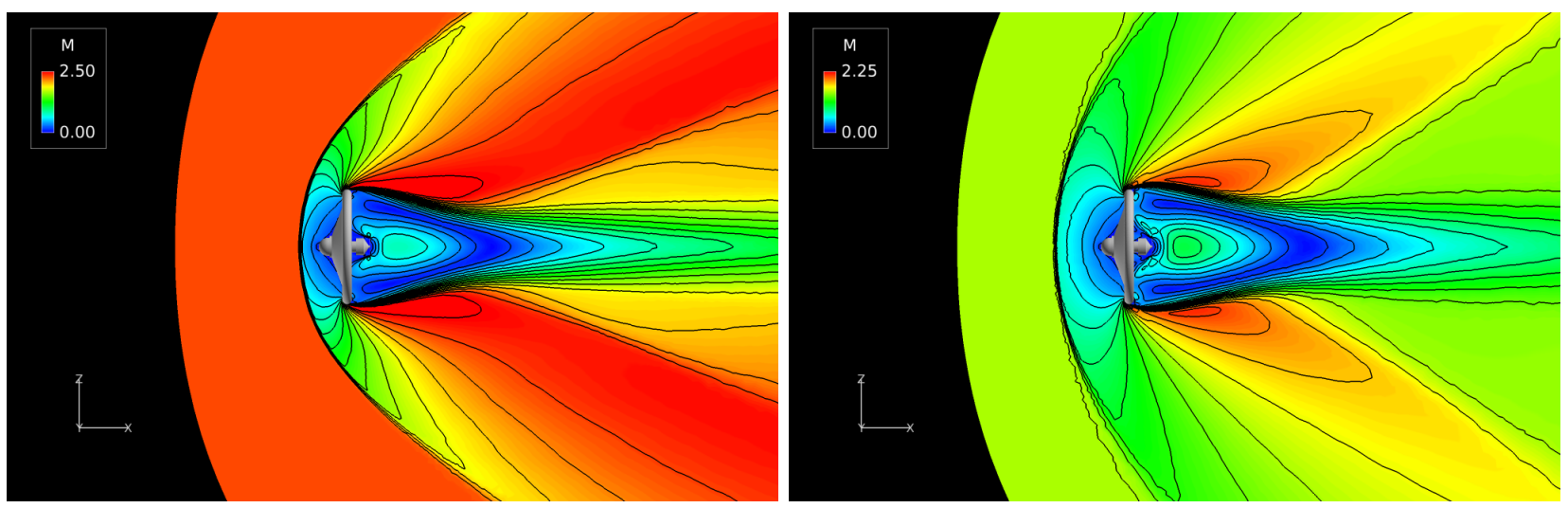

(a) Altitude of $47 \mathrm{~km}\left(\mathrm{Re}=9.01 \times 10^{4}\right.$ and $\left.\mathrm{M}=2.30\right)(\mathrm{b})$ Altitude of $43 \mathrm{~km}\left(\mathrm{Re}=1.00 \times 10^{5}\right.$ and $\left.\mathrm{M}=1.51\right)$

Figure 10: Mach number distribution around SMAAC in the supersonic region.

\subsection{Transonic Flow Region}

In the present transonic region between altitudes of 39 and $37 \mathrm{~km}$, both RG-FaSTAR and FFR are used to predict the aerodynamic characteristics of SMAAC. As is shown in Fig. 8, both RG-FaSTAR and FFR describe a similar trend in which there appears a steep decrease of the drag coefficient in the transonic region. Reasonable analytical solutions related to aerodynamic deceleration are obtained.

The distributions of pressure coefficient around the SMAAC reentry vehicle predicted by RG-FaSTAR (a density-based solver) and FFR (a pressure-based solver) at an altitude of 39 $\mathrm{km}$ are shown in Figs. 11(a) and 11(b), respectively. In addition, Mach number distributions obtained by both solvers are shown in Figs. 12(a) and 12(b). The shock wave disappears with a decrease in the free-stream Mach number. A wake flow is accelerated with expansion near the inflatable torus. The expansion flow becomes weaker as both altitude and Mach number become lower in the transonic region, although the figures are not shown here.

Because in the low-Mach-number approximation one assumes that there are only minor differences in density, it is deduced that the effects of expansion and compression are shown only weakly in the results of FFR compared with the simulation results of RG-FaSTAR at the same altitude. However, because FFR records values for the drag coefficient that are similar to the results of RG-FaSTAR, FFR can also be a useful method in the transonic region for reentry vehicles. The computed result by FFR is basically instantaneous because of the use of LES. However, even though results actually become averaged by the use of the Reynolds-averaged Navier-Stokes equation (in the SST model) in RG-FaSTAR, the flow field is likely to be instantaneous because of its strong unsteadiness.

In this Mach number range, the unsteadiness of the flow field in the wake of SMAAC becomes stronger than the computed results for the supersonic flow region. On the other hand, the flow field in the stagnation region in the vehicle's front is close to being almost steady. The wake structures of the results obtained by these solvers based on their different analytic models are very similar to each other. Small vortices are periodically generated with flow separation near the inflatable torus. A key to predicting the decelerator performance in the transonic region is expected to be resolving both the compressible effect in the front and the wake structure in the rear of this kind of inflatable reentry vehicle. 


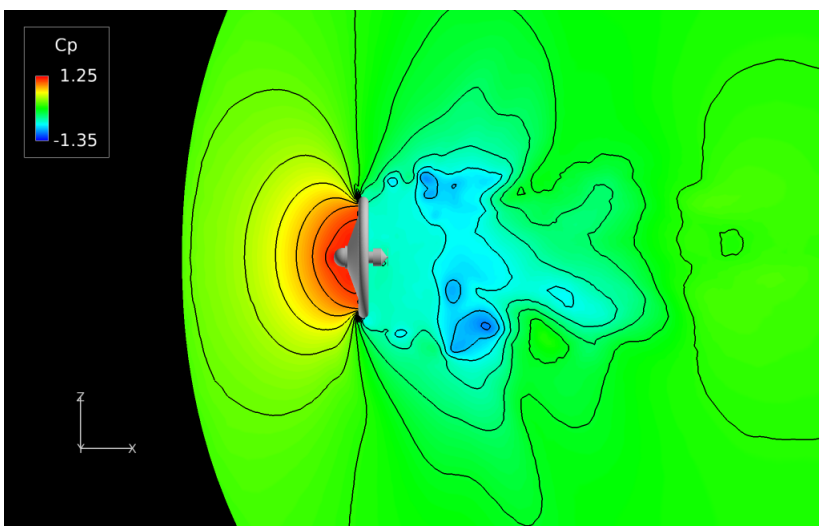

(a) RG-FaSTAR (RANS, SST2003)

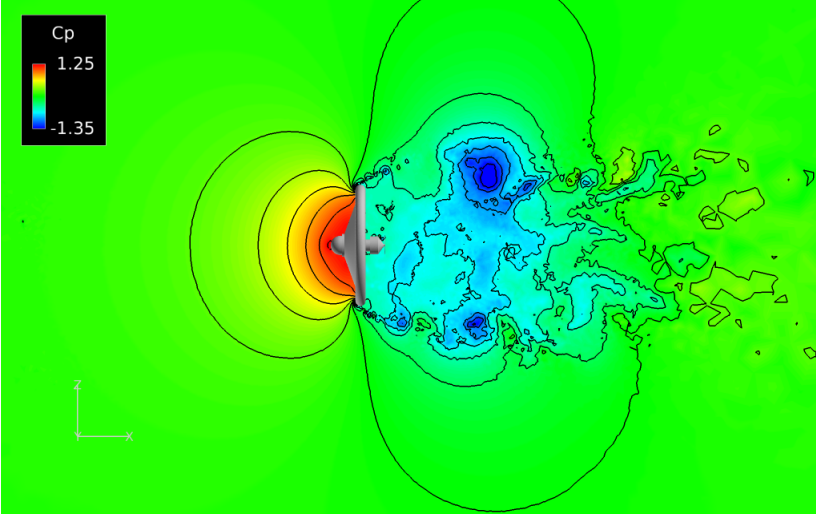

(b) FFR (LES, SS)

Figure 11: Pressure coefficient distribution around SMAAC in the transonic region at an altitude of $39 \mathrm{~km}\left(\mathrm{Re}=1.03 \times 10^{5}\right.$ and $\left.\mathrm{M}=0.871\right)$.

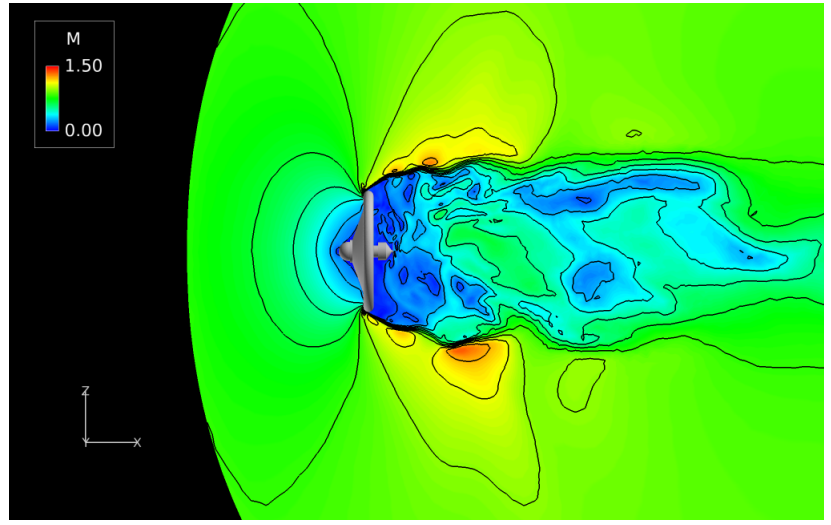

(a) RG-FaSTAR (RANS: SST2003)

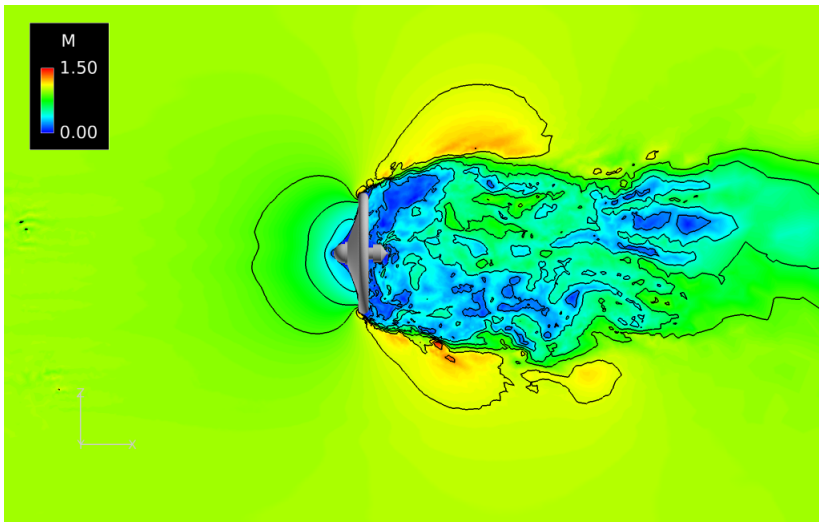

(b) FFR (LES: SS).

Figure 12: Mach number distribution around SMAAC in the transonic region at an altitude of $39 \mathrm{~km}\left(\mathrm{Re}=1.03 \times 10^{5}\right.$ and $\left.\mathrm{M}=0.871\right)$.

\subsection{Subsonic Flow Region}

Below an altitude of $35 \mathrm{~km}\left(\mathrm{Re}=1.31 \times 10^{5}\right.$ and $\left.\mathrm{M}=0.608\right)$, the effect of compressibility becomes weak, whereas the effect of turbulence is relatively enhanced as the Reynolds number becomes high with the decrease in altitude and increase in atmospheric density. In the present subsonic flow region, only FFR is used to simulate such turbulent flows.

The time-averaged pressure coefficient distribution around SMAAC at altitudes of 30 and $10 \mathrm{~km}$ are shown in Figs 13(a) and 13(b), respectively. The vehicle is already sufficiently decelerated at an altitude of $30 \mathrm{~km}$, and then it almost reaches terminal velocity at an altitude of $10 \mathrm{~km}$. From these figures, it is seen that the pressure coefficient at the stagnation point of SMAAC becomes approximately 1.0 at both altitudes. A vortex ring as a time-averaged result is generated in the rear region of the vehicle, and the position of the vortex ring becomes closer to the vehicle with decreasing altitude. The pressure coefficient in the vortex ring is approximately $<-1.5$ because the flow velocity becomes high in the region. The Reynolds number monotonically increases with decreasing altitude during the SMAAC's subsonic flight because the density of air increases faster than the flow velocity decreases by aerodynamic deceleration. The Reynolds number is $\approx 10^{6}$ below an altitude of $5.0 \mathrm{~km}$ and the effect of 
turbulence strongly appears in the flow field.

The distributions of the time-averaged velocity around SMAAC at altitudes of 30 to 10 $\mathrm{km}$ are shown in Figs. 14(a) and 14(b), respectively. Note that the time-averaged velocities are normalized by each free-stream velocity in these figures. For all of the cases in the subsonic region, the velocity of flow decreases in front of SMAAC, and then flow is accelerated and separates near the inflatable torus. Figures 15(a) and 15(b) are the time-averaged velocities near the inflatable torus and surface pressure coefficient at altitudes of 30 and $3 \mathrm{~km}$, respectively. The separation point moves backward for subsonic flow cases as the altitude decreases. This motion of the separation point is one of the reasons for the reduced drag coefficient at lower altitude.
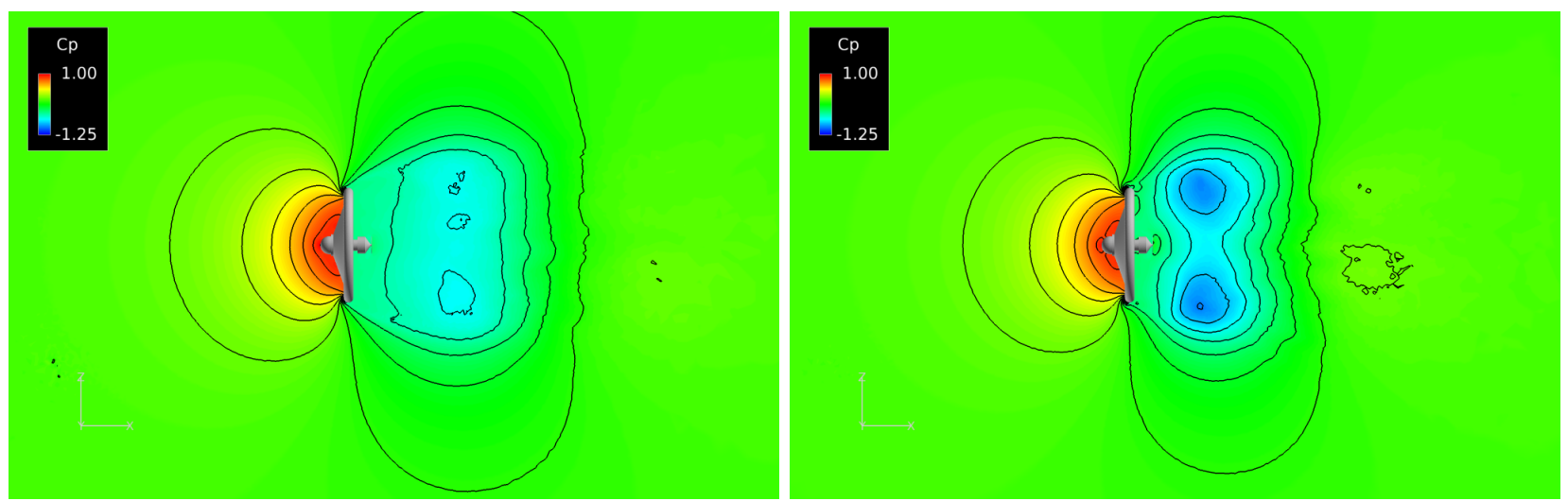

(a) Altitude of $30 \mathrm{~km}\left(\mathrm{Re}=1.87 \times 10^{5}\right.$ and $\left.\mathrm{M}=0.402\right)(\mathrm{b})$ Altitude of $10 \mathrm{~km}\left(\mathrm{Re}=8.18 \times 10^{5}\right.$ and $\left.\mathrm{M}=0.0820\right)$

Figure 13: Time-averaged pressure coefficient distribution around SMAAC in the subsonic region.
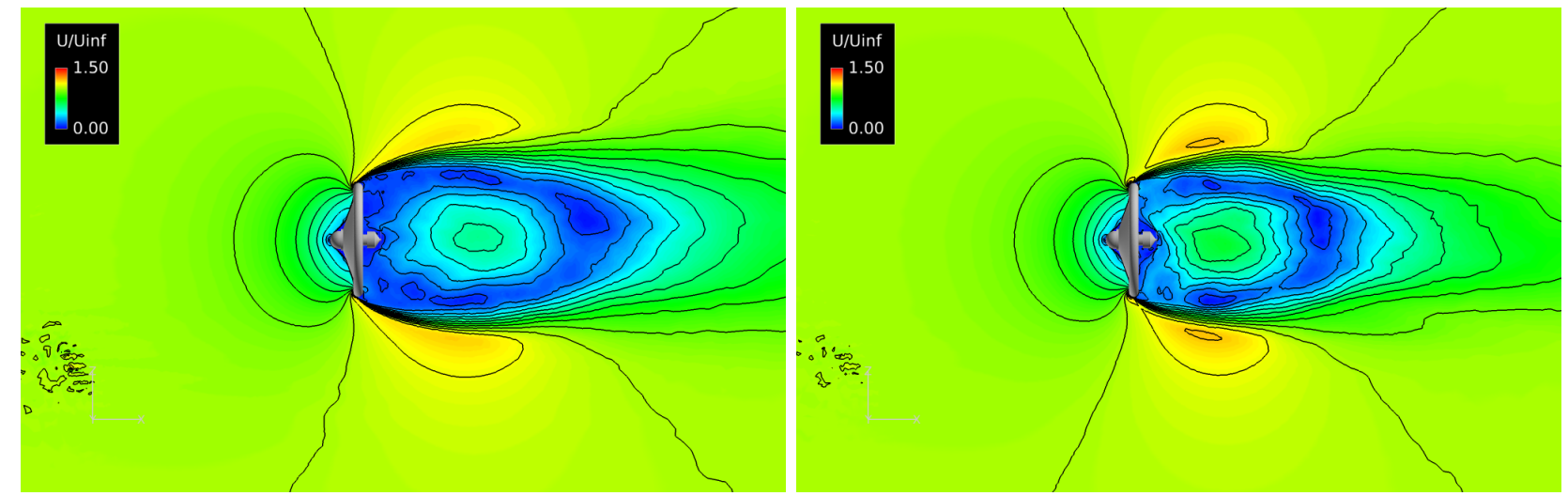

(a) Altitude of $30 \mathrm{~km}\left(\mathrm{Re}=1.87 \times 10^{5}\right.$ and $\left.\mathrm{M}=0.402\right)(\mathrm{b})$ Altitude of $10 \mathrm{~km}\left(\mathrm{Re}=8.18 \times 10^{5}\right.$ and $\left.\mathrm{M}=0.0820\right)$

Figure 14: Time-averaged velocity magnitude distribution, which is normalized by the freestream velocity, around SMAAC in the subsonic region.

The distributions of the instantaneous pressure coefficient around SMAAC at an altitude of $3 \mathrm{~km}$ are shown in Fig. 16. These are computed results obtained over elapsed times of 1.2 to $2.2 \mathrm{~s}$. Figure 17 presents the time history of the total drag coefficient of the SMAAC vehicle at an altitude of $3 \mathrm{~km}$. This figure includes the three components of the drag coefficient: from the capsule, from the membrane aeroshell, and from the inflatable torus. The contribution to the drag from the membrane aeroshell is the largest of the three drag components, because 

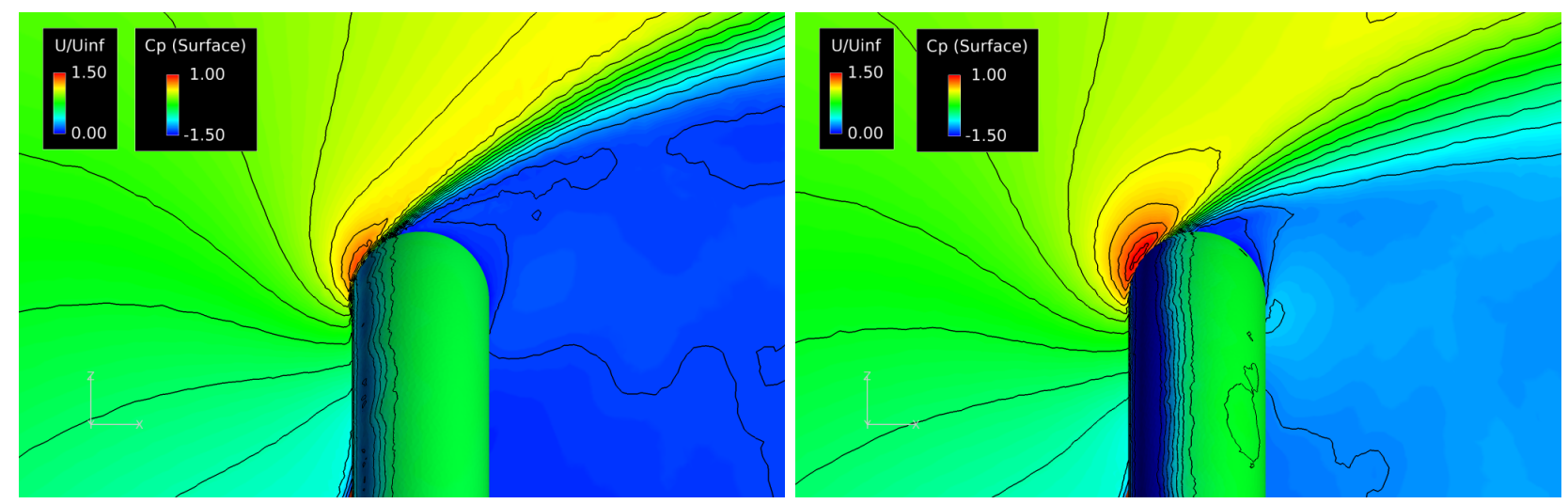

(a) Altitude of $30 \mathrm{~km}\left(\mathrm{Re}=1.87 \times 10^{5}\right.$ and $\left.\mathrm{M}=0.402\right)(\mathrm{b})$ Altitude of $10 \mathrm{~km}\left(\mathrm{Re}=8.18 \times 10^{5}\right.$ and $\left.\mathrm{M}=0.0820\right)$

Figure 15: Separation point movement.

of the large discrepancy between the pressures on the front and rear surfaces of the vehicle. Note that the area of the membrane is also largest in the three components, and this result is reasonable. The drags on the capsule and on the inflatable torus are comparable, although the fluctuation of the torus drag is slightly higher. It is found that the standard deviation of the drag force during 1.0 and $2.25 \mathrm{~s}$ is $\approx 2.5 \%$ of the time-averaged drag at this altitude. The flow field in front of SMAAC almost never changes, maintaining a pressure coefficient of 1.0. However, the fluctuation at the rear side is relatively large. As discussed above with respect to the transonic flow region, small vortices intermittently form near the inflatable torus. In the wake region, these vortices largely grow with a strong negative pressure from the shear stress with the outside flow. This indicates that the variation of the drag force for an inflatable reentry vehicle is mainly attributed to the wake structure in the rear region.

\section{Effect of Membrane Deformation}

The membrane aeroshell of this kind of inflatable reentry vehicle generally deforms by aerodynamic forces during its reentry flight because of its soft material. The changing shape of the aeroshell alters the aerodynamic characteristics including the drag coefficient. Thus, it is necessary to evaluate the effect of membrane deformation to investigate SMAAC's aerodynamic deceleration performance.

\subsection{Deformed Shape}

In this study, membrane deformation of the SMAAC aeroshell is expressed by the virtual particle method as mentioned above. Figures 18 presents an example of the result of membrane deformation for the case of an altitude of $43 \mathrm{~km}$. Initial and deformed shapes are shown in Figs. 18(a) and 18(b), respectively. In addition, each displacement of the membrane particles from the initial shape is described in Fig. 18(b). The aerodynamic force on the aeroshell surface in the membrane deformation model is transposed by that obtained by RG-FaSTAR. It should be noted that the rear of the SMAAC analytical model is flat and is not completely reproduced for simplicity in this section.

The inflatable torus largely moves backward as a result of the aerodynamic force. In addition, the outline of the membrane aeroshell becomes a catenary curve shape. These 


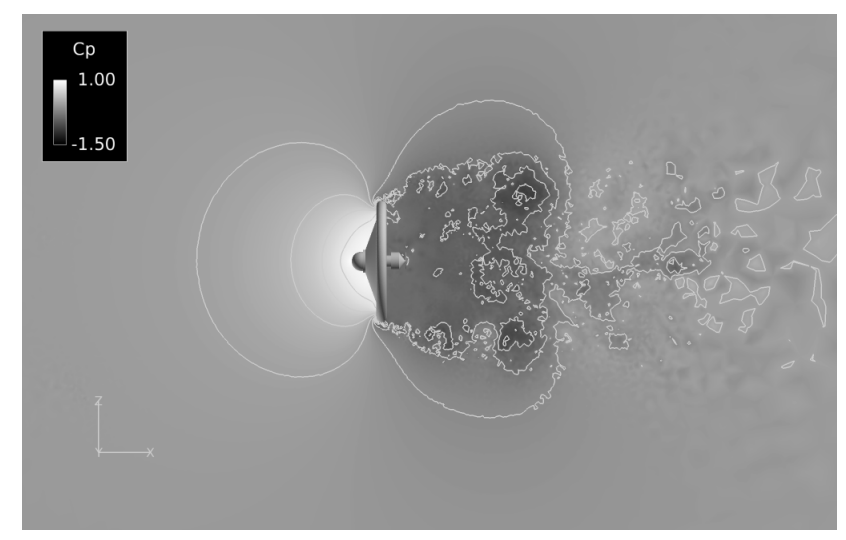

(a) $t=1.2 \mathrm{~s}$

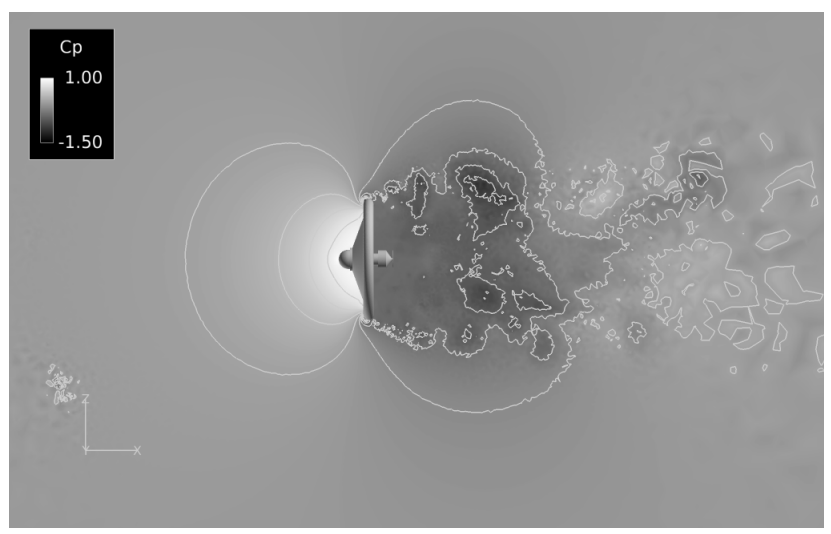

(c) $t=1.6 \mathrm{~s}$

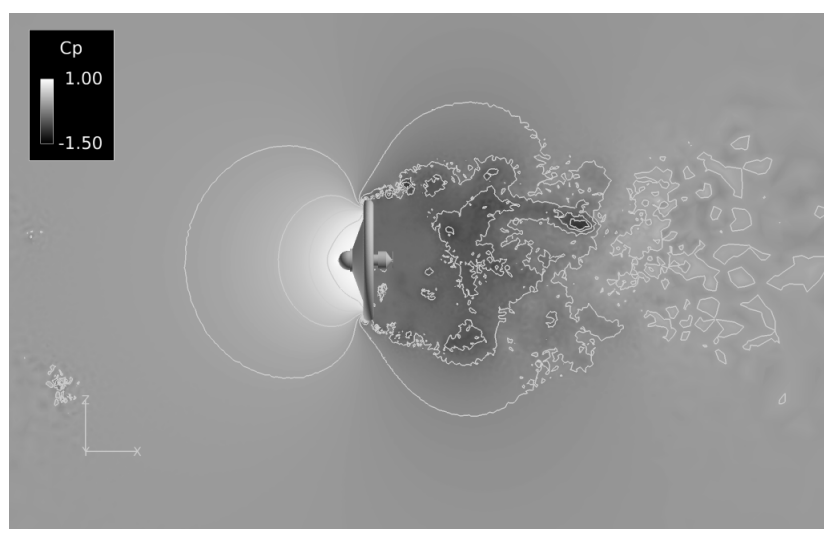

(e) $t=2.0 \mathrm{~s}$

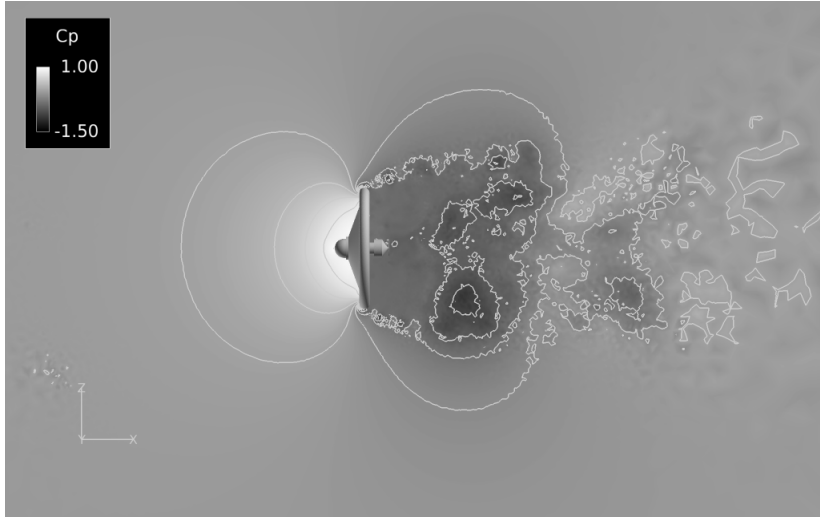

(b) $t=1.4 \mathrm{~s}$

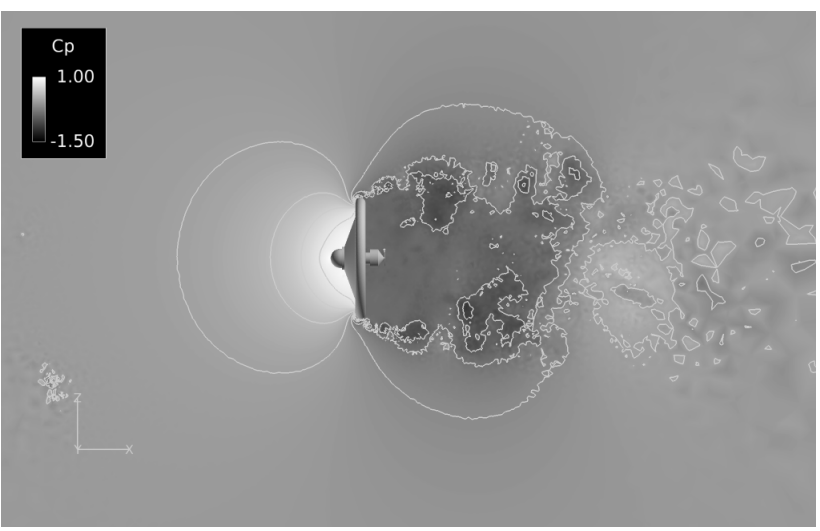

(d) $t=1.8 \mathrm{~s}$

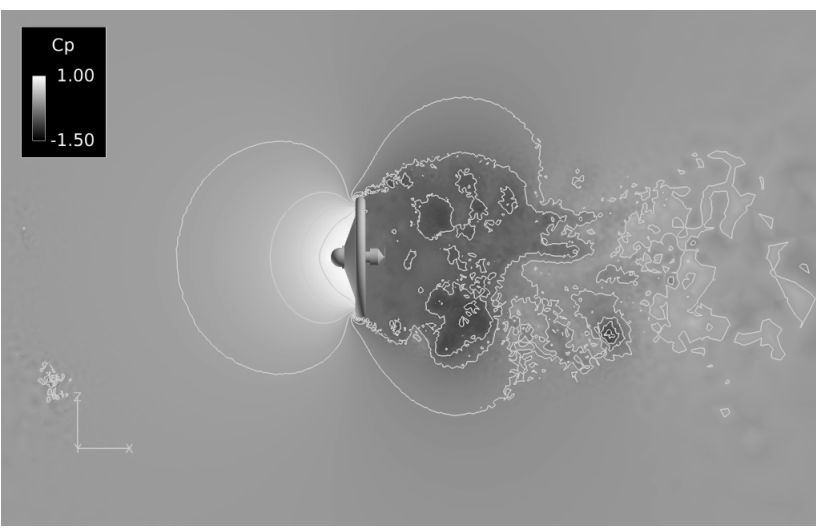

(f) $t=2.2 \mathrm{~s}$

Figure 16: Time series of the instantaneous pressure coefficient around SMAAC at an altitude of $3 \mathrm{~km}\left(\operatorname{Re}=1.08 \times 10^{6}\right.$ and $\left.\mathrm{M}=0.0543\right)$.

changes of the shape are mainly attributed to the elastic force between virtual particles. Several wrinkles are formed on the membrane; these are caused by the bending force.

\subsection{Surface Pressure Distribution}

Figures 19(a) and 19(b) show comparisons of surface pressure coefficients of SMAAC with and without membrane deformation at altitudes of 43 and $10 \mathrm{~km}$, respectively. Cross sections of the initial and deformed models are also shown in these figures. In the supersonic flow 


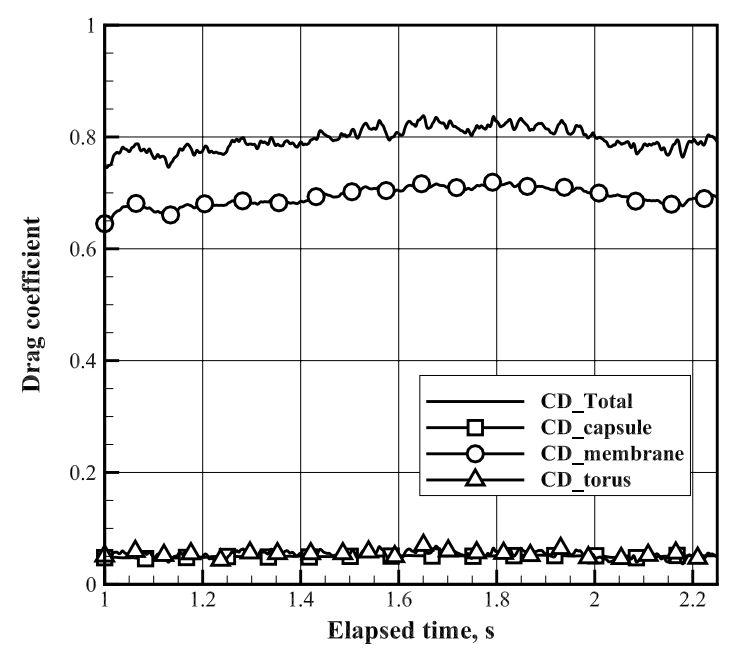

Figure 17: Time history of the drag coefficients at an altitude of $3 \mathrm{~km}\left(\mathrm{Re}=1.08 \times 10^{6}\right.$ and $\mathrm{M}=0.0543)$.

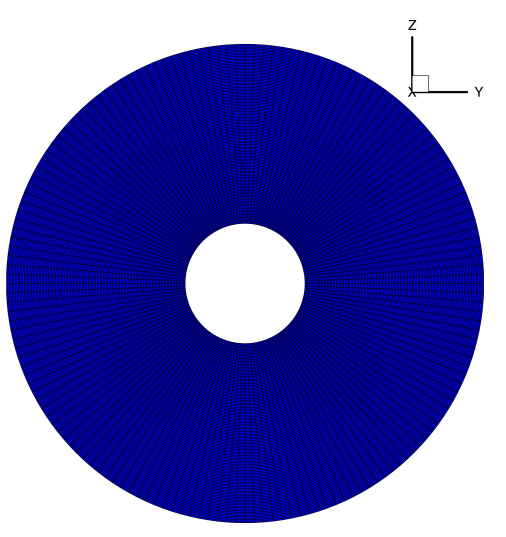

(a) Initial shape

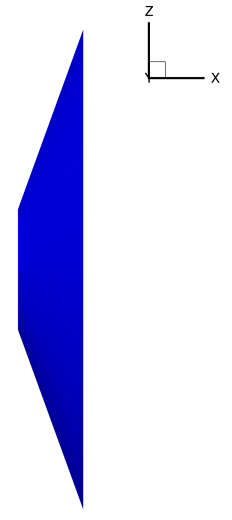$$
\text { e }
$$
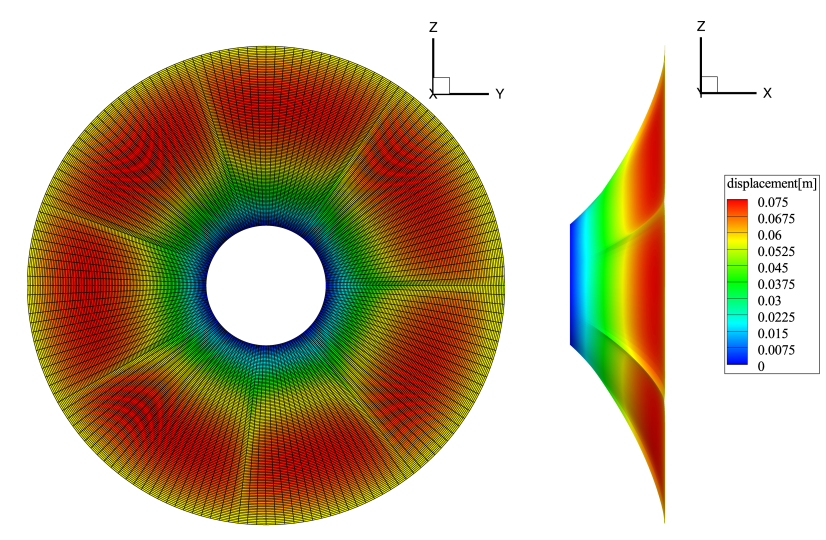

(b) Deformed shape

Figure 18: Example of membrane deformation of SMAAC by the aerodynamic force.

region at an altitude of $43 \mathrm{~km}$, a slight discrepancy of pressures in front of SMAAC with and without a deformation model appears, so that the pressure distribution on the membrane aeroshell becomes flat by deformation. However, the deformation almost never affects the pressure distribution at the rear-side surface. This is because pressure at the front of SMAAC reaches a constant value by formation of normal shock wave and the wake has small influence on pressure at the rear surface. The difference between pressures is also small at the vehicle's front in the subsonic flow region at an altitude of $10 \mathrm{~km}$. In contrast with the case at an altitude of $43 \mathrm{~km}$, the inflatable torus position hardly moves, although the membrane aeroshell is bent. Drag coefficients with and without a deformation model for a flat-rear-type SMAAC are listed in Table 3. As a result, the discrepancy between drags using the rigid and deformed models is small, although aerodynamic coefficients can be strongly affected for a flight case with an angle of attack. This indicates that membrane deformation has a small influence on the aerodynamic decelerator performance in these regions. 


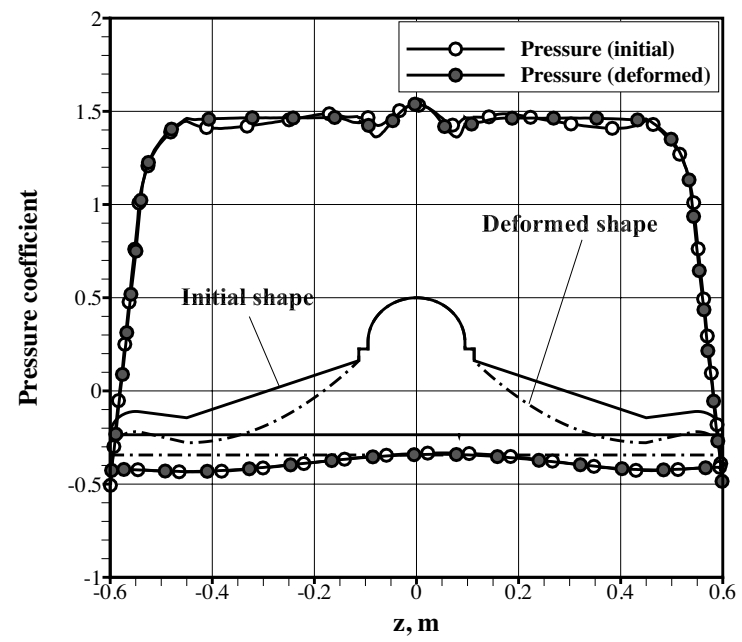

(a) Altitude of $43 \mathrm{~km}$

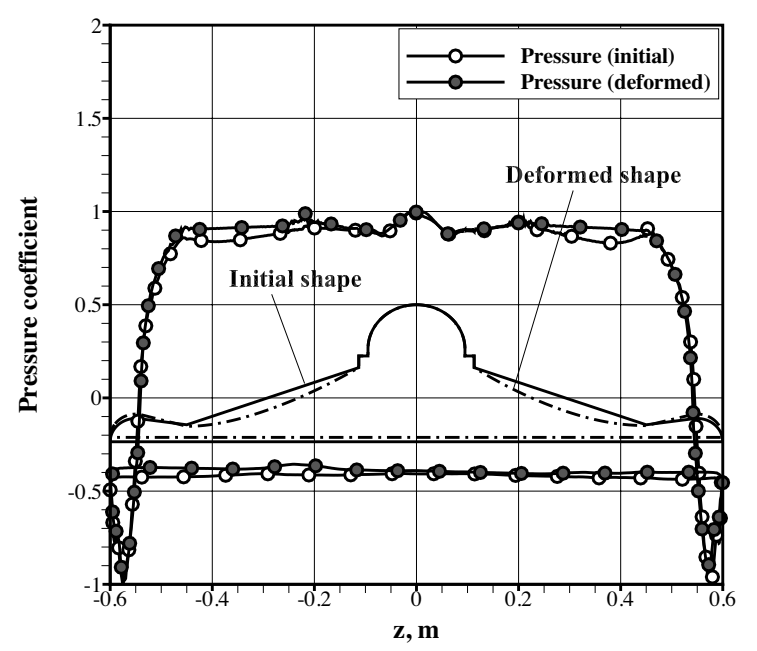

(b) Altitude of $10 \mathrm{~km}$

Figure 19: Comparison of pressure coefficient on the SMAAC surface with and without membrane deformation.

Table 3: Comparison of the drag coefficients with and without a deformation model for SMAAC at altitudes of 43 and $10 \mathrm{~km}$.

\begin{tabular}{ccc}
\hline \hline Altitude, $\mathrm{km}$ & $C_{D}$ (initial) & $C_{D}$ (deformed) \\
\hline 43.0 & 1.59 & 1.59 \\
10.0 & 0.985 & 0.992 \\
\hline \hline
\end{tabular}

\section{Conclusions}

The reentry of an inflatable vehicle lifted to altitude on a sounding rocket was successfully demonstrated. The acceleration and velocity of the vehicle were obtained based on the position measured by the Global Positioning System. Moreover, the drag coefficient during reentry history was evaluated by the measured acceleration and atmosphere profiles from a global reference atmospheric model. The measured drag coefficient was almost a constant value of 1.5 in the supersonic region but gradually decreased to $<1.0$ in the subsonic region as the Mach number decreased. In the transonic region, a steep decrease of the drag coefficient was confirmed.

To investigate the aerodynamics of the inflatable vehicle in detail, numerical simulations of the flow field were performed for various cases between altitudes of 58 and $1.0 \mathrm{~km}$, according to reentry orbit data. Because the flow field widely changes from supersonic to subsonic, the RG-FaSTAR and FFR codes, which are, respectively, compressible and incompressible Navier-Stokes equation solvers, were used to simulate effectively such a range of flow-field conditions. The computed drag coefficient showed good agreement with the measured results. It was clarified that the effect of compressibility in the shock layer in front of the vehicle and a vortex ring in its rear become important mechanisms in the supersonic and subsonic regions, respectively, affecting the prediction of the aerodynamic force in detail.

In addition, flow-field simulations considering membrane deformation based on a particle 
method were also performed. Deformation of the membrane aeroshell and movement of the inflatable torus were confirmed as resulting from the aerodynamic force during reentry. However, it was found that membrane deformation has a relatively small influence on the aerodynamic decelerator performance of this kind of inflatable vehicle.

\section{Acknowledgments}

The sounding rocket experiment was conducted at the Uchinoura Space Center (USC) in collaboration with the Research and Operation Office for Sounding Rocket (ROOSR) at the Japan Aerospace Exploration Agency (JAXA). This research activity was also supported by the Steering Committees for Space Engineering (SCSE). We would like to thank the members of ROOSR, USC, and SCSE for their useful advice and support. This work was supported by JSPS KAKENHI Grant Number 15H04205. The present computational results were obtained using the fast unstructured CFD code FaSTAR, which was developed by JAXA. The computations were mainly performed using the computational facilities (HITACHI HA8000tc/HT210 and FUJITSU PRIMERGY CX400) at the Research Institute for Information Technology, Kyushu University, and the supercomputer system (HITACHI SR16000 model M1) at the Information Initiative Center, Hokkaido University.

\section{References}

[1] M. Gräßilin and U. Schöttle. "Flight Performance Evaluation of the Reentry Mission IRDT-1". In Papers Presented at the 52nd International Astronautical Congress, IAF Paper 01-V305, Toulouse, France, October 1 - 52001.

[2] S.J. Hughes, R.A. Dillman, B.R. Starr, R.A. Stephan, M.C. Lindell, C.J. Player, and D.F.M. Cheatwood. "Inflatable Re-entry Vehicle Experiment (IRVE) Design Overview". AIAA Paper 2005-1636, 2005.

[3] R.R. Rohrschneider and R.D. Braun. "A Survey of Ballute Technology for Aerocapture". Journal of Spacecraft and Rockets, 44(1):10-23, January - February 2007.

[4] P. Reynier and D. Evans. "Postflight Analysis of Inflatable Reentry and Descent Technology Blackout During Earth Reentry". Journal of Spacecraft and Rockets, 46(4):800-809, July-August 2009.

[5] S.J. Hughes, D.F.M. Cheatwood, A.M. Calomino, and H.S. Wright. "Hypersonic Inflatable Aerodynamic Decelerator (HIAD) Technology Development Overview". AIAA Paper 2011-2524, 2011.

[6] T. Abe. "A Self-Consistent Tension Shell Structure for Application to Aerobraking Vehicle and Its Aerodynamic Characteristics". AIAA Paper 1988-3405, 1988.

[7] I.G. Clark, A.L. Hutchings, C.L. Tanner, and R.D. Braun. "Supersonic Inflatable Aerodynamic Decelerators for Use on Future Robotic Missions to Mars". Journal of Spacecraft and Rockets, 46(2):340-352, March - April 2009.

[8] I.G. Clark. Aerodynamic Design, Analysis, and Validation of a Supersonic Inflatable Decelerator. PhD thesis, Georgia Institude of Technology, july 2009. 
[9] C.L. Tanner. Aeroelastic Analysis and Testing of Supersonic Inflatable Aerodynamic Decelerators. PhD thesis, Georgia Institude of Technology, May 2012.

[10] K. Yamada, D. Akita, E. Sato, K. Suzuki, T. Narumi, and T. Abe. "Flare-Type Membrane Aeroshell Flight Test at Free Drop from a Balloon". Journal of Spacecraft and Rockets, 46(3):606-614, May-June 2009.

[11] K. Yamada, T. Abe, K. Suzuki, N. Honma, M. Koyama, Y. Nagata, D. Abe, Y. Kimura, A.K. Hayashi, D. Akita, and H. Makino. "Deployment and Flight Test of Inflatable Membrane Aeroshell using Large Scientific Balloon". AIAA Paper 2011-2579, 2011.

[12] K. Yamada, M. Koyama, Y. Kimura, K. Suzuki, T. Abe, and A.K. Hayashi. "Hypersonic Wind Tunnel Test of a Flare-type Membrane Aeroshell for Atmospheric Entry Capsule". ISTS Special Issue: Selected papers from the 27th ISTS, Transactions of JSASS, 7(ists27):27-32, 2010.

[13] K. Yamada, T. Sonoda, K. Nakashino, and T. Abe. "Structural Strength of Flare-type Membrane Aeroshell Supported by Inflatable Tours against Aerodynamic Force". In Proceedings of 28th International Symposium on Space Technology and Science, ISTS 2011-c-34, Okinawa, Japan, June 5 - 122011.

[14] Y. Takahashi, K. Yamada, and T. Abe. "Examination of Radio Frequency Blackout for an Inflatable Vehicle during Atmospheric Reentry". Journal of Spacecraft and Rockets, 51(2):430-441, March 2014.

[15] K. Yamada, T. Abe, K. Suzuki, O. Imamura, and D. Akita. "Reentry Demonstration Plan of Flare-type Membrane Aeroshell for Atmospheric Entry Vehicle using a Sounding Rocket". AIAA Paper 2011-2521, 2011.

[16] K. Yamada, Y. Nagata, T. Abe, K. Suzuki, O. Imamura, and D. Akita. "Suborbital Reentry Demonstration of Inflatable Flare-Type Thin-Membrane Aeroshell Using a Sounding Rocket". Journal of Spacecraft and Rockets, 52(1):275-284, February-March 2015 .

[17] K. Yamada, Y. Nagata, T. Abe, K. Suzuki, O. Imamura, and D. Akita. "Reentry Demonstration of Flare-type Membrane Aeroshell for Atmospheric Entry Vehicle using a Sounding Rocket". AIAA Paper 2013-1388, 2013.

[18] K. Yamada, Y. Nagata, N. Honma, D. Akita, O. Imamura, T. Abe, and K. Suzuki. "Reentry Demonstration Deployable and Flexible Aeroshell for Atmospheric-Entry Vehicle using Sounding Rocket". In Proceedings of 63th International Astronautical Congress, AC-12-D2.3.3, Naples, Italy, October 1 - 52012.

[19] Y. Nagata, K. Yamada, T. Abe, and k Suzuki. "Attitude Dynamics for Flare-type Membrane Aeroshell Capsule in Reentry Flight Experiment". AIAA Paper 2013-1285, 2013.

[20] Y. Takahashi, K. Yamada, T. Abe, and K. Suzuki. "Aerodynamic Heating around Flaretype Membrane Inflatable Vehicle in Suborbital Reentry Demonstration Flight". Journal of Spacecraft and Rockets, 52(6):1530-1541, Novermber 2015. 
[21] C.G. Justus and D.L. Johnson. "NASA/MSFC Global Reference Atmospheric Model1999 Version (GRAM-99)". NASA TM-1999-209630, May 1999.

[22] D. HA, Y. TAKAHASHI, K. YAMADA, and T. ABE. "Aerodynamic Simulation of Inflatable Re-Entry Vehicle Performance in Low Speed Wind Tunnel". TRANSACTIONS OF THE JAPAN SOCIETY FOR AERONAUTICAL AND SPACE SCIENCES, AEROSPACE TECHNOLOGY JAPAN, 12(ists29):Po_2_57-Po_2_62, 2014.

[23] Y. Takahashi. "Advanced validation of CFD-FDTD combined method using highly applicable solver for reentry blackout prediction". Journal of Physics D: Applied Physics, 49(1):015201, 2016.

[24] N. Oshima. "FrontFlow/red download page". https://www.eng.hokudai.ac.jp/labo/ fluid/download/download.htm.

[25] A. Hashimoto, K. Murakami, T. Aoyama, K. Ishiko, M. Hishida, M. Sakashita, and P.R. Lahur. "Toward the Fastest Unstructured CFD". AIAA Paper 2012-1075, 2012.

[26] E. Shima and K. Kitamura. "Parameter-Free Simple Low-Dissipation AUSM-Family Scheme for All Speeds". AIAA Journal, 49(8):1693-1709, August 2011.

[27] A. Jameson and S. Yoon. "Lower-Upper Implicit Schemes with Multiple Grids for the Euler Equations". AIAA Journal, 25(7):929-935, July 1987.

[28] F.R. Menter, M. Kuntz, and R. Langtry. "Ten Years of Industrial Experience with the SST Turbulence Mode". Turbulence, Heat and Mass Transfer 4, ed: K. Hanjalic, Y. Nagano, and M. Tummers, Begell House, Inc., pages 625-632, 2003.

[29] J. Smagorinsky. "General Circulation Experiments with the Primitive Equations, I. The Basic Experiment". Monthly Weather Review, 91(3):99-164, 1963.

[30] K. Yamada, Y. Kato, and T. Abe. "Numerical Simulation of Hypersonic Flow around Flare-Type Aeroshell with Torus Frame". In Proceedings of $6^{\text {th }}$ Asia Workshop on Computational Fluid Dynamics, Kashiwa, Japan, March 162009. 